W. Czernous and Z. Kamont (Gdańsk)

\title{
COMPARISON OF EXPLICIT AND IMPLICIT DIFFERENCE METHODS FOR QUASILINEAR FUNCTIONAL DIFFERENTIAL EQUATIONS
}

Abstract. We give a theorem on error estimates of approximate solutions for explicit and implicit difference functional equations with unknown functions of several variables. We apply this general result to investigate the stability of difference methods for quasilinear functional differential equations with initial boundary condition of Dirichlet type. We consider first order partial functional differential equations and parabolic functional differential problems. We compare the properties of explicit and implicit difference methods.

We use a comparison technique with nonlinear estimates of Perron type for given functions with respect to the functional variables.

1. Introduction. We are interested in numerical approximation of classical solutions to quasilinear functional differential equations with initial boundary conditions of Dirichlet type. First order partial functional differential equations and parabolic functional differential problems are considered.

Difference schemes for evolution functional differential equations consist in replacing partial derivatives with difference operators. Moreover, because differential equations contain functional variables, some interpolating operators are needed. This leads to difference functional problems which satisfy consistency conditions on classical solutions of original equations. The main task in these considerations is to find difference approximations of functional differential equations which are stable. A comparison technique is used to investigate the stability of functional difference problems.

2010 Mathematics Subject Classification: 65M12, 35R10.

Key words and phrases: functional differential equations, differential and difference inequalities, stability and convergence, interpolating operators. 
It is not our aim here to give a full review of papers concerning explicit difference methods for quasilinear functional differential equations. The papers [3], 4], 18] and the monograph [7] contain such reviews. In recent years, a number of papers on implicit difference methods for functional partial differential equations have been published. Difference approximations of classical solutions to first order partial functional differential equations were considered in 9]. Implicit difference schemes for parabolic equations with initial boundary condition of Dirichlet type were studied in [5], [10]. From the abundant literature concerning the convergence of difference schemes for nonlinear functional differential equations we mention the papers [1], [8], [13], [18]. Monotone iterative methods and implicit difference schemes for computing approximate solutions to parabolic equations with time delays were investigated in [11], [12], [14], [15], [20].

The aim of the present paper is to compare explicit and implicit difference methods for quasilinear functional differential equations.

Two types of assumptions are needed in theorems on convergence of difference methods for evolution functional differential equations. The first type conditions concern regularity of the given functions. The second type conditions concern the mesh. We show that the equations considered here have the following properties. Assumptions on the regularity of the given functions are the same in theorems on convergence of explicit and implicit difference schemes. We prove that assumptions on the mesh are needed for explicit difference methods, but are not necessary for implicit schemes. We show that there are implicit methods which are convergent, while the corresponding explicit schemes are not.

Our results are based on the following idea. Normally, difference inequalities and theorems on recurrent inequalities are used to investigate the stability of explicit and implicit difference schemes. As a rule, these considerations require a lot of calculations so the main properties of the corresponding operators cannot be easily seen. The aim of the present paper is to show that results on explicit and implicit difference schemes are consequences of results on abstract difference equations with an unknown function of several variables.

The authors of [1]-[5], [8]-[15], [18], [20] have assumed that the given functions satisfy the Lipschitz condition or nonlinear estimates of Perron type with respect to the functional variables, and these conditions are global with respect to the functional variables. Our assumptions are more general. We assume that the nonlinear estimates of Perron type are local with respect to the functional variables. It is clear that there are differential equations with deviated variables and differential integral equations such that estimates of Perron type only hold locally. 
We formulate our functional differential problems. For any metric spaces $X$ and $Y$ we denote by $C(X, Y)$ the class of all continuous functions from $X$ into $Y$. We use vectorial inequalities with the understanding that the same inequalities hold between their corresponding components. Let $M_{n \times n}$ be the set of all $n \times n$ matrices with real entries. Write

$E_{0}=\left[-b_{0}, 0\right] \times[-b, b], \quad E=[0, a] \times[-b, b], \quad \partial_{0} E=[0, a] \times([-b, b] \backslash(-b, b))$, where $a>0, b_{0} \in \mathbb{R}_{+}, \mathbb{R}_{+}=[0, \infty)$, and $b=\left(b_{1}, \ldots b_{n}\right), b_{i}>0$ for $i=$ $1, \ldots, n$. For $(t, x) \in E$ we define

$$
D[t, x]=\left\{(\tau, y) \in \mathbb{R}^{1+n}: \tau \leq 0,(t+\tau, x+y) \in E_{0} \cup E\right\} .
$$

It is clear that $D[t, x]=\left[-b_{0}-t, 0\right] \times[-b-x, b-x]$ For a function $z$ : $E_{0} \cup E \rightarrow \mathbb{R}$ and a point $(t, x) \in E$ we define a function $z_{(t, x)}: D[t, x] \rightarrow \mathbb{R}$ by $z_{(t, x)}(\tau, y)=z(t+\tau, x+y),(\tau, y) \in D[t, x]$; that is, we restrict $z$ to the set $\left(E_{0} \cup E\right) \cap\left(\left[-b_{0}, t\right] \times \mathbb{R}^{n}\right)$ and then shift this restriction to the set $D[t, x]$. Write $B=\left[-b_{0}-a, 0\right] \times[-2 b, 2 b]$. Then $D[t, x] \subset B$ for $(t, x) \in E$. Set $\Xi=E \times C(B, \mathbb{R})$ and suppose that

$$
\mathbf{f}: \Xi \rightarrow \mathbb{R}^{n}, \quad \mathbf{f}=\left(f_{1}, \ldots, f_{n}\right), \quad g: \Xi \rightarrow \mathbb{R}, \quad \varphi: E_{0} \cup \partial_{0} E \rightarrow \mathbb{R}
$$

are given functions. Let $z$ be an unknown function of the variables $(t, x)$, $x=\left(x_{1}, \ldots, x_{n}\right)$. We consider the functional differential equation

$$
\partial_{t} z(t, x)=\sum_{i=1}^{n} f_{i}\left(t, x, z_{(t, x)}\right) \partial_{x_{i}} z(t, x)+g\left(t, x, z_{(t, x)}\right)
$$

with the initial boundary condition

$$
z(t, x)=\varphi(t, x) \quad \text { on } E_{0} \cup \partial_{0} E .
$$

We will say that $\mathbf{f}$ and $g$ satisfy the condition $(V)$ if for each $(t, x) \in E$ and for $w, \tilde{w} \in C(B, \mathbb{R})$ such that $w(\tau, y)=\tilde{w}(\tau, y)$ for $(\tau, y) \in D[t, x]$ we have $\mathbf{f}(t, x, w)=\mathbf{f}(t, x, \tilde{w})$ and $g(t, x, w)=g(t, x, \tilde{w})$. The condition $(V)$ means that the values of $\mathbf{f}$ and $g$ at the point $(t, x, w) \in \Xi$ depend on $(t, x)$ and on the restriction of $w$ to the set $D[t, x]$ only. We assume that $\mathbf{f}$ and $g$ satisfy the condition $(V)$ and we consider classical solutions to (1), (2).

Now we formulate initial boundary value problems for parabolic functional differential equations. Suppose that

$$
\begin{aligned}
& \mathbf{F}: \Xi \rightarrow M_{n \times n}, \mathbf{F}=\left[F_{i j}\right]_{i, j=1}^{n}, \\
& \mathbf{G}: \Xi \rightarrow \mathbb{R}^{n}, \quad \mathbf{G}=\left(G_{1}, \ldots, G_{n}\right), \quad G: \Xi \rightarrow \mathbb{R}, \quad \varphi: E_{0} \cup \partial_{0} E \rightarrow \mathbb{R}
\end{aligned}
$$

are given functions. We consider the functional differential equation 


$$
\begin{aligned}
\partial_{t} z(t, x)= & \sum_{i, j=1}^{n} F_{i j}\left(t, x, z_{(t, x)}\right) \partial_{x_{i} x_{j}} z(t, x) \\
& +\sum_{i=1}^{n} G_{i}\left(t, x, z_{(t, x)}\right) \partial_{x_{i}} z(t, x)+G\left(t, x, z_{(t, x)}\right)
\end{aligned}
$$

with the initial boundary condition $(2)$. We assume that $\mathbf{F}, \mathbf{G}, G$ satisfy the following condition $(V)$ : if $(t, x) \in E, w, \tilde{w} \in C(B, \mathbb{R})$ and $w(\tau, y)=\tilde{w}(\tau, y)$ for $(\tau, y) \in D[t, x]$ then $\mathbf{F}(t, x, w)=\mathbf{F}(t, x, \tilde{w}), \mathbf{G}(t, x, w)=\mathbf{G}(t, x, \tilde{w})$, $G(t, x, w)=G(t, x, \tilde{w})$. We consider classical solutions to (3), (2).

The paper is organized as follows. In Section 2 we propose a new method of investigating explicit or implicit difference schemes corresponding to initial boundary value problems for quasilinear functional differential equations. We formulate general difference functional problems with an unknown function of several variables. We give sufficient conditions for the existence and uniqueness of solutions of initial boundary value problems and we prove a theorem on error estimates of approximate solutions. The error is estimated by a solution of an initial problem for a nonlinear difference equation with an unknown function of one variable. In Section 3 we apply the above general results to quasilinear first order partial functional differential equations. Section 4 deals with explicit and implicit difference schemes for parabolic functional differential problems. We use general ideas for finite difference equations which were introduced in the monographs [7], [16], [17].

2. Functional difference equations. For $x \in \mathbb{R}^{n}, W \in M_{n \times n}$ where $x=\left(x_{1}, \ldots, x_{n}\right)$ and $W=\left[w_{i j}\right]_{i, j=1}^{n}$ we put

$$
\|x\|=\sum_{i=1}^{n}\left|x_{i}\right|, \quad\|W\|=\max \left\{\sum_{j=1}^{n}\left|w_{i j}\right|: 1 \leq i \leq n\right\} .
$$

The norm in the space $C(B, \mathbb{R})$ is defined by $\|w\|_{B}=\max \{|w(\tau, y)|$ : $(\tau, y) \in B\}$.

For any sets $X$ and $Y$ we denote by $F(X, Y)$ the set of all functions defined on $X$ and taking values in $Y$. We define a mesh on $E_{0} \cup E$ in the following way. Suppose that $\left(h_{0}, h^{\prime}\right), h^{\prime}=\left(h_{1}, \ldots, h_{n}\right), h_{i}>0$ for $0 \leq i \leq n$, are steps of the mesh. For $h=\left(h_{0}, h^{\prime}\right)$ and $(r, m) \in \mathbb{Z}^{1+n}$ where $m=$ $\left(m_{1}, \ldots, m_{n}\right)$, we define nodal points as follows:

$$
t^{(r)}=r h_{0}, \quad x^{(m)}=\left(x_{1}^{\left(m_{1}\right)}, \ldots, x_{n}^{\left(m_{n}\right)}\right)=\left(m_{1} h_{1}, \ldots, m_{n} h_{n}\right) .
$$

Let $H$ be the set of all $h$ such there are $K_{0} \in \mathbb{Z}$ and $M=\left(M_{1}, \ldots, M_{n}\right) \in \mathbb{N}^{n}$ satisfying $K_{0} h_{0}=b_{0}$ and $\left(M_{1} h_{1}, \ldots, M_{n} h_{n}\right)=b$. Let $K \in \mathbb{N}$ be defined by $K h_{0} \leq a<(K+1) h_{0}$. Write

$$
\mathbb{R}_{h}^{1+n}=\left\{\left(t^{(r)}, x^{(m)}\right):(r, m) \in \mathbb{Z}^{1+n}\right\}
$$


and

$$
\begin{gathered}
E_{0 . h}=E_{0} \cap \mathbb{R}_{h}^{1+n}, \quad E_{h}=E \cap \mathbb{R}_{h}^{1+n}, \quad \partial_{0} E_{h}=\partial_{0} E_{h} \cap \mathbb{R}^{1+n}, \\
E_{h}^{\prime}=\left\{\left(t^{(r)}, x^{(m)}\right) \in E_{h} \backslash \partial_{0} E_{h}: 0 \leq r \leq K-1\right\}, \quad I_{h}=\left\{t^{(r)}: 0 \leq r \leq K\right\} .
\end{gathered}
$$

For $z \in F\left(E_{0 . h} \cup E_{h}, \mathbb{R}\right), \omega \in F\left(I_{h}, \mathbb{R}\right)$ we write $z^{(r, m)}=z\left(t^{(r)}, x^{(m)}\right)$ and $\omega^{(r)}=\omega\left(t^{(r)}\right)$. Set

$$
\begin{gathered}
\Lambda=\left\{\lambda=\left(\lambda_{1}, \ldots, \lambda_{n}\right): \lambda_{i} \in\{-1,0,1\} \text { for } 1 \leq i \leq n \text { and }\|\lambda\| \leq 2\right\}, \\
\Lambda^{\prime}=\Lambda \backslash\{\theta\}, \quad \theta=(0, \ldots, 0) \in \mathbb{R}^{n},
\end{gathered}
$$

and $\chi=1+2 n^{2}$. Note that $\chi$ is the number of elements of $\Lambda$. Let $\psi: \Lambda \rightarrow$ $\{1, \ldots, \chi\}$ be a function such that $\psi(\lambda) \neq \psi(\tilde{\lambda})$ for $\lambda \neq \tilde{\lambda}$. We assume that $\prec$ is an order in $\Lambda$ defined in the following way: if $\psi(\lambda)<\psi(\tilde{\lambda})$ then $\lambda \prec \tilde{\lambda}$. Elements of the space $\mathbb{R}^{\chi}$ will be denoted by $\xi=\left\{\xi_{\lambda}\right\}_{\lambda \in \Lambda}$. Write

$$
A_{h}=\left\{x^{(m)}: m=\left(m_{1}, \ldots, m_{n}\right) \in \Lambda\right\} .
$$

For $\zeta: A_{h} \rightarrow \mathbb{R}$ we put $\zeta^{(m)}=\zeta\left(x^{(m)}\right)$. If $z: E_{0 . h} \cup E_{h} \rightarrow \mathbb{R}$ and $\left(t^{(r)} x^{(m)}\right) \in$ $E_{h} \backslash \partial_{0} E_{h}$ then the function $z_{\langle r, m\rangle}: A_{h} \rightarrow \mathbb{R}$ is defined by

$$
z_{\langle r, m\rangle}(y)=z\left(t^{(r)}, x^{(m)}+y\right), \quad y \in A_{h} .
$$

Solutions of difference functional equations are elements of the space $F\left(E_{0 . h} \cup E_{h}, \mathbb{R}\right)$. Since equations (1) and (3) contain the functional variable $z_{(t, x)}$ which is an element of $C(D[t, x], \mathbb{R})$, we need an interpolating operator $T_{h}: F\left(E_{0 . h} \cup E_{h}, \mathbb{R}\right) \rightarrow C\left(E_{0} \cup E, \mathbb{R}\right)$. Additional assumptions on $T_{h}$ are formulated later in this section. For $z \in F\left(E_{0 . h} \cup E_{h}, \mathbb{R}\right)$ and $\left(t^{(r)}, x^{(m)}\right) \in E_{h}$ we write $\left(T_{h} z\right)_{[r, m]}$ instead of $\left(T_{h} z\right)_{\left(t^{(r)}, x^{(m)}\right)}$. Set $\Omega_{h}=E_{h}^{\prime} \times C(B, \mathbb{R})$ and suppose that

$$
f_{h}: \Omega_{h} \rightarrow \mathbb{R}, \quad G_{h}: \Omega_{h} \rightarrow \mathbb{R}^{\chi}, \quad G_{h}=\left\{G_{h . \lambda}\right\}_{\lambda \in \Lambda}
$$

are given functions. For $(t, x, w) \in \Omega_{h}$ and $\zeta \in F\left(A_{h}, \mathbb{R}\right)$ we put

$$
G_{h}(t, x, w) \circ \zeta=\sum_{\lambda \in \Lambda} G_{h \cdot \lambda}(t, x, w) \zeta^{(\lambda)} .
$$

Let $F_{h}: \Omega_{h} \times F\left(A_{h}, \mathbb{R}\right) \rightarrow \mathbb{R}$ be defined by

$$
F_{h}(t, x, w, \zeta)=f_{h}(t, x, w)+G_{h}(t, x, w) \circ \zeta .
$$

We will say that $f_{h}$ and $G_{h}$ satisfy the condition $(V)$ if for each $(t, x) \in E_{h}^{\prime}$ and $w, \tilde{w} \in C(B, \mathbb{R})$ such that $w(\tau, y)=\tilde{w}(\tau, y)$ for $(\tau, y) \in D[t, x]$ we have $f_{h}(t, x, w)=f(t, x, \tilde{w})$ and $G_{h}(t, x, w)=G_{h}(t, x, \tilde{w})$. For $z \in F\left(E_{0 . h} \cup E_{h}, \mathbb{R}\right)$ and $\left(t^{(r)} x^{(m)}\right) \in E_{h}^{\prime}$ we put

$$
\mathbf{F}_{\text {ex. } h}[z]^{(r, m)}=F_{h}\left(t^{(r)}, x^{(m)},\left(T_{h}\right)_{[r, m]}, z_{\langle r, m\rangle}\right) .
$$

Let $\delta_{0}$ be the difference operator defined by

$$
\delta_{0} z^{(r, m)}=\frac{1}{h_{0}}\left[z^{(r+1, m)}-z^{(r, m)}\right] .
$$


Given $\varphi_{h}: E_{0 . h} \cup \partial_{0} E_{h} \rightarrow \mathbb{R}$, we consider the functional difference equation

$$
\delta_{0} z^{(r, m)}=\mathbf{F}_{\text {ex.h }}[z]^{(r, m)}
$$

with the initial boundary condition

$$
z^{(r, m)}=\varphi_{h}^{(r, m)} \quad \text { on } E_{0 . h} \cup \partial_{0} E_{h} .
$$

If $G_{h}, f_{h}$ satisfy the condition $(V)$ then there exists exactly one solution $u_{h}: E_{0 . h} \cup E_{h} \rightarrow \mathbb{R}$ to (6), (7).

The above problem (6), (7) is obtained in the following way. Explicit difference equations corresponding to (1) or (3) have the form

$$
\delta_{0} z^{(r, m)}=\mathbf{\Psi}_{h}\left(t^{(r)}, x^{(m)}, z(\cdot)\right)
$$

where $\Psi_{h}: E_{h}^{\prime} \times F\left(E_{0 . h} \cup E_{h}, \mathbb{R}\right) \rightarrow \mathbb{R}$ is an operator of Volterra type. Discretization of partial derivatives

$$
\partial_{x} z=\left(\partial_{x_{1}} z, \ldots, \partial_{x_{n}} z\right), \quad \partial_{x x} z=\left[\partial_{x_{i} x_{j}} z\right]_{i, j=1}^{n}
$$

leads to the following observation: the numbers $z^{(r, m+\lambda)}$ where $\lambda \in \Lambda$ appear in the definitions of the difference operators

$$
\delta z=\left(\delta_{1} z, \ldots, \delta_{n} z\right), \quad \delta^{(2)}=\left[\delta_{i j} z\right]_{i, j=1}^{n},
$$

corresponding to these derivatives. It follows that the right hand side of (8) depends on the functional variable $z_{\langle r, m\rangle}$. Since (1) and (3p) contain the functional variable $z_{(t, x)}$ which is an element of $C(D[t, x], \mathbb{R})$ we conclude that $\boldsymbol{\Psi}_{h}$ in $(8)$ depends on $\left(T_{h} z\right)_{[r, m]}$. It is clear that assumptions on the functional variable $\left(T_{h} z\right)_{[r, m]}$ and on $z_{\langle r, m\rangle}$ are not the same in theorems on convergence of difference methods. So it is natural to consider the following explicit difference scheme for (1) or (3):

$$
\delta_{0} z^{(r, m)}=\mathbf{\Phi}_{h}\left(t^{(r)}, x^{(m)},\left(T_{h} z\right)_{[r, m]}, z_{\langle r, m\rangle}\right)
$$

where $\boldsymbol{\Phi}_{h}: \Omega_{h} \times F\left(A_{h}, \mathbb{R}\right) \rightarrow \mathbb{R}$. We associate with 110$)$ the initial boundary condition (7). Equations (1) and (3) are linear with respect to derivatives. It follows that explicit difference schemes for (1), (2) and (3), (2) are linear with respect to difference operators (9). Thus they have the form (6), (7) with $\mathbf{F}_{\text {ex.h }}$ defined by (5). The functions $\varphi_{h}: E_{0 . h} \cup E_{h} \rightarrow \mathbb{R}, h \in H$, approximate $\varphi: E_{0} \cup \partial_{0} E \rightarrow \mathbb{R}$.

Set

$$
\mathbf{F}_{\text {im. } h}[z]^{(r, m)}=F_{h}\left(t^{(r)}, x^{(m)},\left(T_{h}\right)_{[r, m]}, z_{\langle r+1, m\rangle}\right) .
$$

The functional difference equation

$$
\delta_{0} z^{(r, m)}=\mathbf{F}_{\text {im. } h}[z]^{(r, m)}
$$

with the initial boundary condition $(7)$ is considered to be an implicit difference method. 
The above functional difference problems have the property: the numbers $z^{(r+1, m+\lambda)}, \lambda \in \Lambda$, appear in 12 . So 12 , (7) is an implicit functional difference equation. It is important that the functional variable $\left(T_{h} z\right)_{[r, m]}$ appears in (6) and in (12) in the classical sense.

For $z \in C\left(E_{0} \cup E, \mathbb{R}\right)$ and $z_{h} \in F\left(E_{0 . h} \cup E_{h}, \mathbb{R}\right)$ we define the seminorms

$$
\|z\|_{t}=\max \left\{|z(\tau, y)|:(\tau, y) \in\left(E_{0} \cup E\right) \cap\left(\left[-b_{0}, t\right] \times \mathbb{R}^{n}\right)\right\}, \quad 0 \leq t \leq a,
$$
$\left\|z_{h}\right\|_{h . r}=\max \left\{\left|z_{h}(\tau, y)\right|:(\tau, y) \in\left(E_{0 . h} \cup E_{h}\right) \cap\left(\left[-b_{0}, t^{(r)}\right] \times \mathbb{R}^{n}\right)\right\}, 0 \leq r \leq K$.

Assumption $H[\varrho]$. The function $\varrho:[0, a] \times \mathbb{R}_{+} \rightarrow \mathbb{R}_{+}$is continuous and it is nondecreasing with respect to both variables and for each $\eta \in \mathbb{R}_{+}$there exists on $[0, a]$ the maximal solution of the Cauchy problem

$$
\omega^{\prime}(t)=\varrho(t, \omega(t)), \quad \omega(0)=\eta .
$$

Assumption $H\left[G_{h}, f_{h}\right]$. The functions $G_{h}: \Omega_{h} \rightarrow \mathbb{R}^{\chi}, f_{h}: \Omega_{h} \rightarrow \mathbb{R}$ satisfy the condition $(V)$ and

1) for $(t, x, w) \in \Omega_{h}$ we have

$$
G_{h . \lambda}(t, x, w) \geq 0 \quad \text { for } \lambda \in \Lambda^{\prime} \quad \text { and } \quad \sum_{\lambda \in \Lambda} G_{h . \lambda}(t, x, w) \leq 0,
$$

2) there is $\varrho:[0, a] \times \mathbb{R}_{+} \rightarrow \mathbb{R}_{+}$such that Assumption $H[\varrho]$ is satisfied and

$$
\left|f_{h}(t, x, w)\right| \leq \varrho\left(t,\|w\|_{B}\right) \quad \text { for }(t, x, w) \in \Omega_{h}, h \in H,
$$

3) there is $\tilde{\eta} \in \mathbb{R}_{+}$such that

$$
\left|\varphi_{h}^{(r, m)}\right| \leq \tilde{\eta} \quad \text { on } E_{0 . h} \quad \text { and } \quad\left|\varphi_{h}^{(r, m)}\right| \leq \omega\left(t^{(r)}, \tilde{\eta}\right) \quad \text { on } \partial_{0} E_{h}
$$

where $\omega(\cdot, \tilde{\eta})$ is the maximal solution to 13 for $\eta=\tilde{\eta}$.

Assumption $H\left[T_{h}\right]$. The operator $T_{h}: F\left(E_{0 . h} \cup E_{h}, \mathbb{R}\right) \rightarrow C\left(E_{0} \cup E, \mathbb{R}\right)$ satisfies the conditions:

1) for $z, \bar{z} \in F\left(E_{0 . h} \cup E_{h}, \mathbb{R}\right)$ we have

$$
\left\|T_{h}[z]-T_{h}[\bar{z}]\right\|_{t^{(r)}} \leq\|z-\bar{z}\|_{h . r}, \quad 0 \leq r \leq K,
$$

2) if $z: E_{0} \cup E \rightarrow \mathbb{R}_{+}$is of class $C^{1}$ then there is $\gamma_{\star}: H \rightarrow \mathbb{R}_{+}$such that

$$
\left\|T_{h}\left[z_{h}\right]-z\right\|_{t} \leq \gamma_{\star}(h), \quad 0 \leq t \leq a, \quad \lim _{h \rightarrow 0} \gamma_{\star}(h)=0,
$$

where $z_{h}$ is the restriction of $z$ to $E_{0 . h} \cup E_{h}$,

$3)$ if $\mathbf{0}_{h} \in F\left(E_{0 . h} \cup E_{h}, \mathbb{R}\right)$ is given by $\mathbf{0}_{h}(t, x)=0$ for $(t, x) \in E_{0 . h} \cup E_{h}$ then $T_{h}\left[\mathbf{0}_{h}\right](t, x)=0$ for $(t, x) \in E_{0} \cup E$.

An example of the operator $T_{h}$ satisfying Assumption $H\left[T_{h}\right]$ can be found in [7, Chapter 5]. We begin with a theorem on the existence and estimates of solutions to (6), (7) and (12), (7). 
TheOREm 2.1. Suppose that Assumptions $H\left[G_{h}, f_{h}\right]$ and $H\left[T_{h}\right]$ are satisfied. Then:

I. There exists exactly one solution $v_{h}: E_{0 . h} \cup E_{h} \rightarrow \mathbb{R}$ to (12), (7) and

$$
\left|v_{h}^{(r, m)}\right| \leq \omega\left(t^{(r)}, \tilde{\eta}\right) \quad \text { on } E_{h} .
$$

II. If additionally the steps of the mesh satisfy the condition

$$
1+h_{0} G_{h . \theta}(t, x, w) \geq 0 \quad \text { on } \Omega_{h},
$$

then there is exactly one solution $u_{h}: E_{0 . h} \cup E_{h} \rightarrow \mathbb{R}$ to (6), (7) and

$$
\left|u_{h}^{(r, m)}\right| \leq \omega\left(t^{(r)}, \tilde{\eta}\right) \quad \text { on } E_{h} .
$$

Proof. I. Suppose that $0 \leq r<K$ is fixed and that the solution $v_{h}$ to (12), (7) is given on $\left(E_{0 . h} \cup E_{h}\right) \cap\left(\left[-b_{0}, t^{(r)}\right] \times \mathbb{R}^{n}\right)$. We prove that the values $v_{h}^{(r+1, m)},-M \leq m \leq M$, exist and are unique. It is sufficient to show that there exists exactly one solution of the linear system

$$
\begin{aligned}
& z^{(r+1, m)}=v_{h}^{(r, m)}+h_{0} F_{h}\left(t^{(r)}, x^{(m)},\left(T_{h} v_{h}\right)_{[r, m]}, z_{\langle r+1, m\rangle}\right) \\
& \text { for }\left(t^{(r)}, x^{(m)}\right) \in E_{h}^{\prime},
\end{aligned}
$$

where $F_{h}$ is given by (4). It follows from (14) that the homogeneous system corresponding to (18), (19) has exactly one zero solution. Thus the solution $v_{h}$ is given on $\left(E_{0 . h} \cup E_{h}\right) \cap\left(\left[-b_{0}, t^{(r+1)}\right] \times \mathbb{R}^{n}\right)$ and it is unique. Since $v_{h}$ is given on $E_{0 . h}$, the proof of the existence and uniqueness of a solution to (12), (7) is completed by induction with respect to $r, 0 \leq r \leq K$.

Now we prove (15). We conclude from (12) that

$$
\begin{aligned}
v_{h}^{(r+1, m)}[1 & \left.+h_{0} G_{h . \theta}\left(t^{(r)}, x^{(m)},\left(T_{h} v_{h}\right)_{[r, m]}\right)\right] \\
= & v_{h}^{(r+1, m)}+h_{0} \sum_{\lambda \in \Lambda^{\prime}} G_{h . \lambda}\left(t^{(r)}, x^{(m)},\left(T_{h} v_{h}\right)_{[r, m]}\right) v_{h}^{(r+1, m+\lambda)} \\
& \quad+h_{0} f_{h}\left(t^{(r)}, x^{(m)},\left(T_{h} v_{h}\right)_{[r, m]}\right)
\end{aligned}
$$

where $\left(t^{(r)}, x^{(m)}\right) \in E_{h}^{\prime}$. Let $\omega_{h}: I_{h} \rightarrow \mathbb{R}_{+}$be defined by $\omega_{h}^{(r)}=\left\|v_{h}\right\|_{h . r}$, $0 \leq r \leq K$. It follows from condition 2) of Assumption $H\left[G_{h}, f_{h}\right]$ and from (14), 20) that

$$
\omega_{h}^{(r+1)} \leq \omega_{h}^{(r)}+h_{0} \varrho\left(t^{(r)}, \omega_{h}^{(r)}\right) \quad \text { for } 0 \leq r \leq K-1
$$

and $\omega_{h}^{(r)} \leq \tilde{\eta}$. The function $\omega(\cdot, \tilde{\eta})$ satisfies the recurrent inequality

$$
\omega\left(t^{(r+1)}, \tilde{\eta}\right) \geq \omega\left(t^{(r)}, \tilde{\eta}\right)+h_{0} \varrho\left(t^{(r)}, \omega\left(t^{(r)}, \tilde{\eta}\right)\right), \quad 0 \leq r \leq K-1 .
$$

Since $\omega_{h}^{(0)} \leq \omega\left(t^{(0)}, \tilde{\eta}\right)$, we have $\omega_{h}^{(r)} \leq \omega\left(t^{(r)}, \tilde{\eta}\right)$ for $0 \leq r \leq K$ and 15 follows. 
II. It is clear that there exists exactly one solution $u_{h}: E_{0 . h} \cup E_{h} \rightarrow \mathbb{R}$ to (6), (7). We conclude from (6) that

$$
\begin{aligned}
& u_{h}^{(r+1, m)}=u_{h}^{(r, m)}\left[1+h_{0} G_{h . \theta}\left(t^{(r)}, x^{(m)},\left(T_{h} u_{h}\right)_{[r, m]}\right)\right] \\
& +h_{0} \sum_{\lambda \in \Lambda^{\prime}} G_{h . \lambda}\left(t^{(r)}, x^{(m)},\left(T_{h} u_{h}\right)_{[r, m]}\right) u_{h}^{(r, m+\lambda)}+h_{0} f_{h}\left(t^{(r)}, x^{(m)},\left(T_{h} u_{h}\right)_{[r, m]}\right)
\end{aligned}
$$

where $\left(t^{(r)}, x^{(m)}\right) \in E_{h}^{\prime}$. Let $\tilde{\omega}_{h}: I_{h} \rightarrow \mathbb{R}_{+}$be defined by $\tilde{\omega}_{h}^{(r)}=\left\|u_{h}\right\|_{h . r}$, $0 \leq r \leq K$. It follows from condition 2) of Assumption $H\left[G_{h}, f_{h}\right]$ and from (14), (16), 22, that

$$
\tilde{\omega}_{h}^{(r+1)} \leq \tilde{\omega}_{h}^{(r)}+h_{0} \varrho\left(t^{(r)}, \tilde{\omega}_{h}^{(r)}\right) \quad \text { for } 0 \leq r \leq K-1,
$$

and $\tilde{\omega}_{h}^{(0)} \leq \tilde{\eta}$. The above relations and 21 imply 17 . This completes the proof of the theorem.

We will consider approximate solutions to (6), (7) and (12), (7). Suppose that the functions $z_{h}: E_{0 . h} \cup E_{h} \rightarrow \mathbb{R}, h \in H$, and $\alpha_{0}, \gamma: H \rightarrow \mathbb{R}_{+}$satisfy

$$
\left|\delta_{0} z_{h}^{(r, m)}-\mathbf{F}_{\text {ex.h }}\left[z_{h}\right]^{(r, m)}\right| \leq \gamma(h) \quad \text { on } E_{h}^{\prime}
$$

and

$$
\begin{aligned}
\left|z_{h}^{(r, m)}-\varphi_{h}^{(r, m)}\right| & \leq \alpha_{0}(h) \quad \text { on } E_{0 . h} \cup \partial_{0} E_{h}, \\
\lim _{h \rightarrow 0} \alpha_{0}(h) & =0, \quad \lim _{h \rightarrow 0} \gamma(h)=0 .
\end{aligned}
$$

The functions $z_{h}, h \in H$, satisfying the above relations are considered to be approximate solutions to (6), (7). If

$$
\left|\delta_{0} z_{h}^{(r, m)}-\mathbf{F}_{\text {im. } h}\left[z_{h}\right]^{(r, m)}\right| \leq \gamma(h) \quad \text { on } E_{h}^{\prime}
$$

and conditions (24), (25) are satisfied then $z_{h}, h \in H$, are approximate solutions to (12), (7).

We give a theorem on estimates of differences between the exact and approximate solutions to (6), (7) and (12), (7). Suppose that Assumptions $H\left[G_{h}, f_{h}\right]$ and $H\left[T_{h}\right]$ are satisfied. Write $C=\omega(a, \tilde{\eta})$ and

$$
K_{C(B, \mathbb{R})}[\tilde{C}]=\left\{w \in C(B, \mathbb{R}):\|w\|_{B} \leq \tilde{C}\right\} .
$$

Assumption $H_{0}[\sigma]$. The function $\sigma:[0, a] \times \mathbb{R}_{+} \rightarrow \mathbb{R}_{+}$satisfies the conditions:

1) $\sigma$ is continuous and it is nondecreasing with respect to both variables,

2) $\sigma(t, 0)=0$ for $t \in[0, a]$ and the maximal solution of the Cauchy problem

$$
\omega^{\prime}(t)=\sigma(t, \omega(t)), \quad \omega(0)=0,
$$

is $\tilde{\omega}(t)=0$ for $t \in[0, a]$. 
Theorem 2.2. Suppose that Assumptions $H\left[G_{h}, f_{h}\right]$ and $H\left[T_{h}\right]$ are satisfied and there exist $\sigma:[0, a] \times \mathbb{R}_{+} \rightarrow \mathbb{R}_{+}$and $Y_{h} \subset F\left(A_{h}, \mathbb{R}\right)$ such that Assumption $H_{0}[\sigma]$ is satisfied and for $w, \tilde{w} \in K_{C(B, \mathbb{R})}[\tilde{C}]$ and $\zeta \in Y_{h}$ we have

$$
\left|F_{h}(t, x, w, \zeta)-F_{h}(t, x, \tilde{w}, \zeta)\right| \leq \sigma\left(t,\|w-\tilde{w}\|_{B}\right), \quad(t, x) \in E_{h}^{\prime} .
$$

I. Suppose that $v_{h}: E_{0 . h} \cup E_{h} \rightarrow \mathbb{R}$ is a solution to (12), (7) and $\tilde{v}_{h}$ : $E_{0 . h} \cup E_{h} \rightarrow \mathbb{R}$ satisfies the conditions:

(i) $\left\|\tilde{v}_{h}\right\|_{h . r} \leq \tilde{C}$ and $\left(\tilde{v}_{h}\right)_{\langle r, m\rangle} \in Y_{h}$ for $0 \leq r \leq K$,

(ii) conditions (24)-26) are satisfied for $z_{h}=\tilde{v}_{h}$.

Then there is $\alpha: H \rightarrow \mathbb{R}_{+}$such that

$$
\left|\left(v_{h}-\tilde{v}_{h}\right)^{(r, m)}\right| \leq \alpha(h) \quad \text { on } E_{h} \quad \text { and } \quad \lim _{h \rightarrow 0} \alpha(h)=0 .
$$

II. Suppose that the steps of the mesh satisfy (16) and $u_{h}: E_{0 . h} \cup E_{h} \rightarrow \mathbb{R}$ is a solution to (6), (7) and $\tilde{u}_{h}: E_{0 . h} \cup E_{h} \rightarrow \mathbb{R}$ satisfies the conditions:

(i) $\left\|\tilde{u}_{h}\right\|_{h . r} \leq \tilde{C}$ and $\left(\tilde{u}_{h}\right)_{\langle r, m\rangle} \in Y_{h}$ for $0 \leq r \leq K$,

(ii) conditions 23)-25) are satisfied with $z_{h}=\tilde{u}_{h}$.

Then there is $\alpha: H \rightarrow \mathbb{R}_{+}$such that

$$
\left|\left(u_{h}-\tilde{u}_{h}\right)^{(r, m)}\right| \leq \alpha(h) \quad \text { on } E_{h} \quad \text { and } \quad \lim _{h \rightarrow 0} \alpha(h)=0 .
$$

Proof. I. The existence of the solution $v_{h}$ to (12), (7) follows from Theorem 2.1. Let $\Gamma_{\mathrm{im} . h}: E_{h}^{\prime} \rightarrow \mathbb{R}$ be defined by

$$
\delta_{0} \tilde{v}_{h}^{(r, m)}=\mathbf{F}_{\mathrm{im} . h}\left[\tilde{v}_{h}\right]^{(r, m)}+\Gamma_{\mathrm{im} . h}^{(r, m)} .
$$

Then $\left|\Gamma_{\mathrm{im} . h}^{r, m)}\right| \leq \gamma(h)$ on $E_{h}^{\prime}$. It follows from 12 that

$$
\begin{aligned}
\left(\tilde{v}_{h}-v_{h}\right)^{(r+1, m)}\left[1-h_{0} G_{h . \theta}\left(t^{(r)}, x^{(m)},\left(T_{h} v_{h}\right)_{[r, m]}\right)\right] \\
=\left(\tilde{v}_{h}-v_{h}\right)^{(r, m)} \\
\quad+h_{0} \sum_{\lambda \in \Lambda^{\prime}} G_{h . \lambda}\left(t^{(r)}, x^{(m)},\left(T_{h} v_{h}\right)_{[r, m]}\right)\left(\tilde{v}_{h}-v_{h}\right)^{(r, m+\lambda)}+\Gamma_{\mathrm{im} . h}^{(r, m)} \\
\quad+F_{h}\left(t^{(r)}, x^{(m)},\left(T_{h} \tilde{v}_{h}\right)_{[r, m]},\left(\tilde{v}_{h}\right)_{\langle r+1, m\rangle}\right) \\
\quad-F_{h}\left(t^{(r)}, x^{(m)},\left(T_{h} v_{h}\right)_{[r, m]},\left(\tilde{v}_{h}\right)_{\langle r+1, m\rangle}\right) .
\end{aligned}
$$

Let $\varepsilon_{h}: I_{h} \rightarrow \mathbb{R}_{+}$be given by $\varepsilon_{h}^{(r)}=\left\|\tilde{v}_{h}-v_{h}\right\|_{h . r}, 0 \leq r \leq K$. We conclude from Assumptions $H\left[G_{h}, f_{h}\right], H\left[T_{h}\right]$ and $H[\sigma]$ and from (30) that

$$
\varepsilon_{h}^{(r+1)} \leq \varepsilon_{h}^{(r)}+h_{0} \sigma\left(t^{(r)}, \varepsilon_{h}^{(r)}\right)+h_{0} \gamma(h), \quad 0 \leq r \leq K-1,
$$

and $\varepsilon_{h}^{(0)} \leq \alpha_{0}(h)$. Denote by $\omega(\cdot, h)$ the maximal solution of the Cauchy problem

$$
\omega^{\prime}(t)=\sigma(t, \omega(t))+\gamma(h), \quad \omega(0)=\alpha_{0}(h) .
$$


It follows that $\omega(\cdot, h)$ is defined on $[0, a]$ and $\lim _{h \rightarrow 0} \omega(t, h)=0$ uniformly on $[0, a]$. Moreover we have

$$
\omega\left(t^{(r+1)}, h\right) \geq \omega\left(t^{(r)}, h\right)+h_{0} \sigma\left(t^{(r)}, \omega\left(t^{(r)}, h\right)\right)+h_{0} \gamma(h), \quad 0 \leq r \leq K-1 .
$$

This gives $\varepsilon_{h}^{(r)} \leq \omega\left(t^{(r)}, h\right)$ for $0 \leq r \leq K$ and condition (28) is satisfied with $\alpha(h)=\omega(a, h)$.

II. It is clear that there exists a solution $u_{h}$ to (6), (7). Let $\Gamma_{\text {ex.h }}: E_{h}^{\prime} \rightarrow \mathbb{R}$ be defined by

$$
\delta_{0} \tilde{v}_{h}^{(r, m)}=\mathbf{F}_{\text {ex.h } h}\left[\tilde{v}_{h}\right]^{(r, m)}+\Gamma_{\text {ex.h }}^{(r, m)} .
$$

Then $\left|\Gamma_{\text {ex.h }}^{r, m)}\right| \leq \gamma(h)$ on $E_{h}^{\prime}$. It follows from (6) that

$$
\begin{array}{r}
\left(\tilde{v}_{h}-v_{h}\right)^{(r+1, m)}=\left(\tilde{v}_{h}-v_{h}\right)^{(r, m)}\left[1+h_{0} G_{h . \theta}\left(t^{(r)}, x^{(m)},\left(T_{h} u_{h}\right)_{[r, m]}\right)\right] \\
+h_{0} \sum_{\lambda \in \Lambda^{\prime}} G_{h . \lambda}\left(t^{(r)}, x^{(m)},\left(T_{h} u_{h}\right)_{[r, m]}\right)\left(\tilde{u}_{h}-u_{h}\right)^{(r, m+\lambda)}+\Gamma_{\mathrm{im} . h}^{(r, m)} \\
+F_{h}\left(t^{(r)}, x^{(m)},\left(T_{h} \tilde{u}_{h}\right)_{[r, m]},\left(\tilde{u}_{h}\right)_{\langle r, m\rangle}\right)-F_{h}\left(t^{(r)}, x^{(m)},\left(T_{h} u_{h}\right)_{[r, m]},\left(\tilde{u}_{h}\right)_{\langle r, m\rangle}\right) .
\end{array}
$$

Let $\varepsilon_{h}: I_{h} \rightarrow \mathbb{R}_{+}$be given by $\varepsilon_{h}^{(r)}=\left\|\tilde{u}_{h}-u_{h}\right\|_{h . r}, 0 \leq r \leq K$. We conclude from Assumptions $H\left[G_{h}, f_{h}\right], H\left[T_{h}\right]$ and $H[\sigma]$ and from (16), (33) that the function $\varepsilon_{h}$ satisfies 31 and $\varepsilon_{h}^{(0)} \leq \alpha_{0}(h)$. Thus condition 290 is satisfied with $\alpha(h)=\omega(a, h)$ where $\omega(\cdot, h)$ is the maximal solution to (32).

This completes the proof of the theorem.

REMARK 2.3. Suppose that all the assumptions of Theorem 2.2 hold with $\sigma(t, p)=L p$ on $[0, a] \times \mathbb{R}_{+}$where $L \in \mathbb{R}_{+}$, so the operator $F_{h}$ satisfies the Lipschitz condition with respect to the functional variable on $E_{h}^{\prime} \times K_{C(B, \mathbb{R})}[\tilde{C}] \times Y_{h}$. Then $\left|\left(v_{h}-\tilde{v}_{h}\right)^{(r, m)}\right| \leq \tilde{\alpha}(h)$ on $E_{h}$ and $\left|\left(u_{h}-\tilde{v}_{h}\right)^{(r, m)}\right|$ $\leq \tilde{\alpha}(h)$ on $E_{h}$ where

$$
\begin{array}{ll}
\tilde{\alpha}(h)=\alpha_{0}(h) e^{L a}+\gamma(h) \frac{e^{L a}-1}{L} & \text { if } L>0, \\
\tilde{\alpha}(h)=\alpha_{0}(h)+a \gamma(h) & \text { if } L=0 .
\end{array}
$$

The above estimates are obtained by solving problem 32 with $\sigma(t, p)=L p$.

It follows that we have obtained the same estimates of errors for the implicit and for the explicit difference equations.

3. First order partial functional differential equations. We formulate difference methods for (1), (2). Consider the operator $U_{h}: \Omega_{h} \times F\left(A_{h}, \mathbb{R}\right)$ $\rightarrow \mathbb{R}$ defined in the following way. Let $\left(t^{(r)}, x^{(m)}, w, \zeta\right) \in \Omega_{h} \times F\left(A_{h}, \mathbb{R}\right)$. Write

$$
\begin{aligned}
& J_{+}^{(r, m)}[w]=\left\{i \in\{1, \ldots, n\}: f_{i}\left(t^{(r)}, x^{(m)}, w\right) \geq 0\right\} \\
& J_{-}^{(r, m)}[w]=\{1, \ldots, n\} \backslash J_{+}^{(r, m)}[w]
\end{aligned}
$$


and

$$
U_{h}\left(t^{(r)}, x^{(m)}, w, \zeta\right)=\sum_{i=1}^{n} f_{i}\left(t^{(r)}, x^{(m)}, w\right) \delta_{j} \zeta^{(\theta)}+g\left(t^{(r)}, x^{(m)}, w\right) .
$$

The expressions $\left(\delta_{1} \zeta^{(\theta)}, \ldots, \delta_{n} \zeta^{(\theta)}\right)$ are given in the following way. Set $e_{i}=$ $(0, \ldots, 0,1,0, \ldots, 0) \in \mathbb{R}^{n}$ with 1 in the $i$ th place and

$$
\begin{array}{ll}
\delta_{j} \zeta^{(\theta)}=\frac{1}{h_{j}}\left[\zeta^{\left(e_{j}\right)}-\zeta^{(\theta)}\right] & \text { for } j \in J_{+}^{(r, m)}[w], \\
\delta_{j} \zeta^{(\theta)}=\frac{1}{h_{j}}\left[\zeta^{(\theta)}-\zeta^{\left(-e_{j}\right)}\right] & \text { for } j \in J_{-}^{(r, m)}[w] .
\end{array}
$$

For $z \in F\left(E_{0 . h} \cup E_{h}, \mathbb{R}\right)$ and $\left(t^{(r)}, x^{(m)}\right) \in E_{h}^{\prime}$ we write

$$
\begin{aligned}
\mathbb{U}_{\text {ex.h }}[z]^{(r, m)} & =U_{h}\left(t^{(r)}, x^{(m)},\left(T_{h} z\right)_{[r, m]}, z_{\langle r, m\rangle}\right), \\
\mathbb{U}_{\text {im. } h}[z]^{(r, m)} & =U_{h}\left(t^{(r)}, x^{(m)},\left(T_{h} z\right)_{[r, m]}, z_{\langle r+1, m\rangle}\right) .
\end{aligned}
$$

Given $\varphi_{h}: E_{0 . h} \cup E_{h} \rightarrow \mathbb{R}$, we approximate the classical solution to (1), (2) with the solution of the functional difference equation

$$
\delta_{0} z^{(r, m)}=\mathbb{U}_{\text {ex.h }}[z]^{(r, m)}
$$

with the initial boundary condition

$$
z^{(r, m)}=\varphi_{h}^{(r, m)} \quad \text { on } E_{0 . h} \cup \partial_{0} E_{h} .
$$

The difference equation

$$
\delta_{0} z^{(r, m)}=\mathbb{U}_{\text {im.h }}[z]^{(r, m)}
$$

with initial boundary condition (37) is considered to be an implicit difference scheme for (1), 22).

Assumption $H_{0}[\mathbf{f}, g, \varphi]$. The functions $\mathbf{f}: \Xi \rightarrow \mathbb{R}^{n}, g: \Xi \rightarrow \mathbb{R}$ and $\varphi: E_{0} \cup \partial_{0} E \rightarrow \mathbb{R}$ are such that

1) $\mathbf{f}$ and $g$ are continuous and satisfy the condition $(V)$,

2) there is $\varrho:[0, a] \times \mathbb{R}_{+} \rightarrow \mathbb{R}_{+}$such that of Assumption $H[\varrho]$ holds and

$$
|g(t, x, w)| \leq \varrho\left(t,\|w\|_{B}\right) \quad \text { on } \Xi
$$

3) $\varphi \in C\left(E_{0} \cup \partial_{0} E, \mathbb{R}\right), \varphi_{h} \in F\left(E_{0 . h} \cup \partial_{0} E_{h}, \mathbb{R}\right)$ and there is $\alpha_{0}: H \rightarrow \mathbb{R}_{+}$ such that

$$
\left|\varphi(t, x)-\varphi_{h}(t, x)\right| \leq \alpha_{0}(h) \quad \text { on } E_{0 . h} \cup \partial_{0} E_{h} \quad \text { and } \quad \lim _{h \rightarrow 0} \alpha_{0}(h)=0,
$$

4) the constant $\tilde{\eta} \in \mathbb{R}_{+}$is defined by the relations

$$
\begin{aligned}
|\varphi(t, x)| \leq \tilde{\eta} \quad \text { on } E_{0}, \quad\left|\varphi_{h}(t, x)\right| \leq \tilde{\eta} \quad \text { on } E_{0 . h}, \\
|\varphi(t, x)| \leq \omega(t, \tilde{\eta}) \quad \text { on } \partial_{0} E, \quad\left|\varphi_{h}(t, x)\right| \leq \omega(t, \tilde{\eta}) \quad \text { on } \partial_{0} E_{h},
\end{aligned}
$$

where $\omega(\cdot, \tilde{\eta})$ is the maximal solution to 13 for $\eta=\tilde{\eta}$. 
We give estimates of solutions to (1), (2).

Lemma 3.1. If Assumption $H_{0}[\mathbf{f}, g, \varphi]$ is satisfied and $\tilde{z}: E_{0} \cup E \rightarrow \mathbb{R}$ is a solution to (1), (2) and $\tilde{z}$ is of class $C^{1}$ then

$$
|\tilde{z}(t, x)| \leq \omega(t, \tilde{\eta}) \quad \text { on } E .
$$

Proof. For $\varepsilon>0$ we denote by $\omega(\cdot, \tilde{\eta}, \varepsilon)$ the maximal solution of the Cauchy problem

$$
\omega^{\prime}(t)=\varrho(t, \omega(t))+\varepsilon, \quad \omega(0)=\tilde{\eta}+\varepsilon .
$$

Then $\omega(\cdot, \tilde{\eta}, \varepsilon)$ is defined on $[0, a]$ and

$$
\lim _{\varepsilon \rightarrow 0} \omega(t, \tilde{\eta}, \varepsilon)=\omega(t, \tilde{\eta}) \quad \text { uniformly on }[0, a] .
$$

Write $\tilde{\omega}(t)=\|\tilde{z}\|_{t}, t \in[0, a]$. We prove that

$$
\tilde{\omega}(t)<\omega(t, \tilde{\eta}, \varepsilon) \quad \text { for } t \in[0, a] .
$$

Suppose for contradiction that assertion (44) fails to be true. Then there is $(\tilde{t}, \tilde{x}) \in(0, a] \times(-b, b)$ such that

$$
\tilde{\omega}(t)<\omega(t, \tilde{\eta}, \varepsilon) \quad \text { for } t \in[0, \tilde{t}) \quad \text { and } \quad \tilde{\omega}(\tilde{t})=\omega(\tilde{t}, \tilde{\eta}, \varepsilon)=|\tilde{z}(\tilde{t}, \tilde{x})| \text {. }
$$

Two cases are possible: either (i) $\tilde{z}(\tilde{t}, \tilde{x})=\omega(\tilde{t}, \tilde{\eta}, \varepsilon)$ or (ii) $\tilde{z}(\tilde{t}, \tilde{x})=-\omega(\tilde{t}, \tilde{\eta}, \varepsilon)$. In the first case,

$$
D_{-} \tilde{\omega}(\tilde{t}) \geq \omega^{\prime}(\tilde{t}, \tilde{\eta}, \varepsilon)
$$

where $D_{-}$is the left hand lower Dini derivative. Since $\partial_{x} \tilde{z}(\tilde{t}, \tilde{x})=\theta$, we have

$$
D_{-} \tilde{\omega}(\tilde{t}) \leq \partial_{t} \tilde{z}(\tilde{t}, \tilde{x})=g\left(\tilde{t}, \tilde{x}, \tilde{z}_{(\tilde{t}, \tilde{x})}\right) \leq \varrho(\tilde{t}, \omega(\tilde{t}, \tilde{\eta}, \varepsilon))<\omega^{\prime}(\tilde{t}, \tilde{\eta}, \varepsilon),
$$

which contradicts $(45)$. The case $\tilde{z}(\tilde{t}, \tilde{x})=-\omega(\tilde{t}, \tilde{\eta}, \varepsilon)$ can be treated in a similar way. Thus (44) is proved. Letting $\varepsilon$ tend to zero in (44) we obtain (41). This completes the proof.

Lemma 3.2. Suppose that Assumption $H\left[T_{h}\right]$ and $H_{0}[\mathbf{f}, g, \varphi]$ are satisfied. Then:

I. There exists exactly one solution $v_{h}: E_{0 . h} \cup E_{h} \rightarrow \mathbb{R}$ to (38), (37), and

$$
\left|v_{h}^{(r, m)}\right| \leq \omega\left(t^{(r)}, \tilde{\eta}\right) \quad \text { on } E_{h} .
$$

II. Moreover, if

$$
1-h_{0} \sum_{i=1}^{n} \frac{1}{h_{i}}\left|f_{i}(t, x, w)\right| \geq 0 \quad \text { on } \Xi
$$

then the solution $u_{h}: E_{0 . h} \cup E_{h} \rightarrow \mathbb{R}$ to (36), 37) satisfies the condition

$$
\left|u_{h}^{(r, m)}\right| \leq \omega\left(t^{(r)}, \tilde{\eta}\right) \quad \text { on } E_{h} .
$$


Proof. We apply Theorem 2.1. Define $G_{h}: \Omega_{h} \rightarrow \mathbb{R}^{\chi}, G_{h}=\left\{G_{h . \lambda}\right\}_{\lambda \in \Lambda}$, and $f_{h}: \Omega_{h} \rightarrow \mathbb{R}$ in the following way. Let $\left(t^{(r)}, x^{(m)}, w\right) \in \Omega_{h}$. Write

$$
\begin{aligned}
& \Lambda_{+}^{(r, m)}[w]=\left\{\lambda \in \Lambda: \text { there is } j \in J_{+}^{(r, m)}[w] \text { such that } \lambda=e_{j}\right\}, \\
& \Lambda_{-}^{(r, m)}[w]=\left\{\lambda \in \Lambda: \text { there is } j \in J_{-}^{(r, m)}[w] \text { such that } \lambda=-e_{j}\right\} .
\end{aligned}
$$

Set

$$
\begin{aligned}
f_{h}\left(t^{(r)}, x^{(m)}, w\right) & =g\left(t^{(r)}, x^{(m)}, w\right), \\
G_{h . \theta}\left(t^{(r)}, x^{(m)}, w\right) & =-\sum_{j=1}^{n} \frac{1}{h_{j}}\left|f_{j}\left(t^{(r)}, x^{(m)}, w\right)\right|, \\
G_{h . e_{j}}\left(t^{(r)}, x^{(m)}, w\right) & =\frac{1}{h_{j}} f_{j}\left(t^{(r)}, x^{(m)}, w\right) \quad \text { for } j \in J_{+}^{(r, m)}[w], \\
G_{h .-e_{j}}\left(t^{(r)}, x^{(m)}, w\right) & =-\frac{1}{h_{j}} f_{j}\left(t^{(r)}, x^{(m)}, w\right) \quad \text { for } j \in J_{-}^{(r, m)}[w], \\
G_{h . \lambda}\left(t^{(r)}, x^{(m)}, w\right) & =0 \quad \text { for } \lambda \in \Lambda \backslash\left[\Lambda_{i .+}^{(r, m)}[w] \cup \Lambda_{i .-}^{(r, m)}[w] \cup\{\theta\}\right] .
\end{aligned}
$$

Then Assumption $H\left[G_{h}\right]$ is satisfied and problems (36), (37) and (38), (37) are equivalent to (6), (7) and (12), (7) respectively. Our lemma follows from Theorem 2.1.

REMARK 3.3. The stability of difference equations generated by hyperbolic systems of conservation laws is strictly connected with the so-called Courant-Friedrichs-Levy (CFL) conditions (see [6, Chapter III]). The (CFL) conditions for the quasilinear equation (1) have the form (46).

Suppose that Assumptions $H\left[T_{h}\right]$ and $H_{0}[\mathbf{f}, g, \varphi]$ are satisfied. Write $\tilde{C}=$ $\omega(a, \tilde{\eta})$ and

$$
X[\tilde{C}]=\left\{w \in C(B, \mathbb{R}):\|w\|_{B} \leq \tilde{C}\right\} .
$$

Assumption $H[\sigma]$. The function $\sigma:[0, a] \times \mathbb{R}_{+} \rightarrow \mathbb{R}_{+}$satisfies:

1) $\sigma$ is continuous and it is nondecreasing with respect to both variables,

2) $\sigma(t, 0)=0$ for $t \in[0, a]$ and for each $c \geq 1$ the maximal solution of the Cauchy problem

$$
\omega^{\prime}(t)=c \sigma(t, \omega(t)), \quad \omega(0)=0,
$$

is $\tilde{\omega}(t)=0$ for $t \in[0, a]$.

Assumption $H[\mathbf{f}, g, \varphi]$. The functions $\mathbf{f}: \Xi \rightarrow \mathbb{R}^{n}$ and $g: \Xi \rightarrow \mathbb{R}, \varphi:$ $E_{0} \cup \partial_{0} E \rightarrow \mathbb{R}$ satisfy Assumption $H_{0}[\mathbf{f}, g, \varphi]$ and there is $\sigma:[0, a] \times \mathbb{R}_{+} \rightarrow \mathbb{R}_{+}$ such that Assumption $H[\sigma]$ is satisfied and

$$
\begin{aligned}
\| \mathbf{f}(t, x, w)-\mathbf{f}(t, x, \tilde{w} \| & \leq \sigma\left(t,\|w-\tilde{w}\|_{B}\right), \\
\mid g(t, x, w)-g(t, x, \tilde{w} \mid & \leq \sigma\left(t,\|w-\tilde{w}\|_{B}\right),
\end{aligned}
$$

where $(t, x) \in E, w, \tilde{w} \in X[\tilde{C}]$. 
REMARK 3.4. It is important that we have assumed inequalities (48), 49 for $w, \tilde{w} \in X[\tilde{C}]$. It is clear that there are differential integral equations and differential equations with deviated variables such that Assumption $H[\mathbf{f}, g, \sigma]$ holds and estimates (48), (49) are not satisfied on $\Xi$.

TheOREM 3.5. Suppose that Assumptions $H\left[T_{h}\right]$ and $H[\mathbf{f}, g, \varphi]$ are satisfied and $\tilde{z}: E_{0} \cup E \rightarrow \mathbb{R}$ is a solution to $(1),(2)$ and $\tilde{z}$ is of class $C^{1}$ and $\tilde{z}_{h}$ is the restriction of $\tilde{z}$ to the set $E_{0 . h} \cup E_{h}$. Then:

I. There exists exactly one solution $v_{h}: E_{0 . h} \cup E_{h} \rightarrow \mathbb{R}$ to (38), (37) and there is $\alpha: H \rightarrow \mathbb{R}_{+}$such that

$$
\left|\left(v_{h}-\tilde{z}_{h}\right)^{(r, m)}\right| \leq \alpha(h) \quad \text { on } E_{h} \quad \text { and } \quad \lim _{h \rightarrow 0} \alpha(h)=0 .
$$

II. If condition (46) is satisfied then there is $\alpha: H \rightarrow \mathbb{R}_{+}$such that

$$
\left|\left(u_{h}-\tilde{z}_{h}\right)^{(r, m)}\right| \leq \alpha(h) \quad \text { on } E_{h} \quad \text { and } \quad \lim _{h \rightarrow 0} \alpha(h)=0,
$$

where $u_{h}: E_{0 . h} \cup E_{h} \rightarrow \mathbb{R}$ is a solution to (36), (37).

Proof. We apply Theorem 2.2 to prove (50) and (51). Let $\tilde{c} \in \mathbb{R}_{+}$be such that

$$
\left\|\partial_{x} \tilde{z}(t, x)\right\| \leq \tilde{c} \quad \text { on } E .
$$

Denote by $Y_{h}$ the class of all $\zeta \in F\left(A_{h}, \mathbb{R}\right)$ such that

$$
\left|\frac{1}{h_{j}}\left[\zeta^{\left(e_{j}\right)}-\zeta^{(\theta)}\right]\right| \leq \tilde{c}, \quad\left|\frac{1}{h_{j}}\left[\zeta^{(\theta)}-\zeta^{\left(-e_{j}\right)}\right]\right| \leq \tilde{c}, \quad j=1, \ldots, n .
$$

Then we have

$$
\left\|\tilde{z}_{h}\right\|_{h . r} \leq \tilde{C} \quad \text { and } \quad\left(\tilde{z}_{h}\right)_{\langle r, m\rangle} \in Y_{h} \quad \text { for } 0 \leq r \leq K .
$$

It follows from Assumption $H[\mathbf{f}, g, \varphi]$ that for $w, \tilde{w} \in X_{h}[\bar{C}]$ and $\zeta \in Y_{h}$ we have

$$
\left|U_{h}(t, x, w, \zeta)-U_{h}(t, x, \tilde{w}, \zeta)\right| \leq(1+\tilde{c}) \sigma\left(t,\|w-\tilde{w}\|_{B}\right), \quad(t, x) \in E_{h}^{\prime} .
$$

It is clear that condition (46) for equation (36) is equivalent to 15 for (6). Thus all the assumptions of Theorem 2.2 are satisfied and assertions (50), (51) follow.

REMARK 3.6. Suppose that all the assumptions of Theorem 3.5 are satisfied with $\sigma(t, p)=\tilde{L} p$ on $[0, a] \times \mathbb{R}_{+}$where $\tilde{L} \in \mathbb{R}_{+}$. Then there is $L \in \mathbb{R}_{+}$ such that $\left|\left(\tilde{z}_{h}-v_{h}\right)^{(r, m)}\right| \leq \tilde{\alpha}(h)$ on $E_{h}$ and $\left|\left(\tilde{z}_{h}-u_{h}\right)^{(r, m)}\right| \leq \tilde{\alpha}(h)$ on $E_{h}$ where $\tilde{\alpha}: H \rightarrow \mathbb{R}_{+}$is given by (34), 35).

Now we present numerical examples. Put $n=2$. Solutions of the initial boundary value problems below are defined on $E=[0,0.5] \times[-1,1] \times[-1,1]$. 
Consider the differential equation with deviated variables

$$
\begin{aligned}
\partial_{t} z & (t, x, y) \\
= & 4 x\{1+\cos [z(t, 0.25(x+\sqrt{3} y), 0.25(\sqrt{3} x-y))-z(t, 0.5 x, 0.5 y)]\} \partial_{x} z(t, x, y) \\
& +4 y\{1-\cos [z(t, 0.25 \sqrt{2}(x-y), 0.25 \sqrt{2}(x+y))-z(t, 0.5 x, 0.5 y)]\} \partial_{y} z(t, x, y) \\
& +z(t, x, y)\left(x^{2}+y^{2}-1-16 x^{2} t\right)
\end{aligned}
$$

with the initial boundary conditions

$$
\begin{gathered}
z(0, x, y)=1, \quad(x, y) \in[-1,1] \times[-1,1], \\
z(t,-1, y)=z(t, 1, y)=e^{t y^{2}}, \quad t \in[0,0.5], y \in[-1,1], \\
z(t, x,-1)=z(t, x, 1)=e^{t x^{2}}, \quad t \in[0,0.5], x \in[-1,1] .
\end{gathered}
$$

The solution of the above problem is $v(t, x, y)=\exp \left[t\left(x^{2}+y^{2}-1\right)\right]$. The following tables show maximal values of errors for several step sizes.

Table 1. Errors for explicit Euler method

\begin{tabular}{cccc}
\hline$h_{0}$ & $h_{1}=h_{2}$ & Maximal error & Time $[\mathrm{s}]$ \\
\hline $2^{-8}$ & $2^{-4}$ & $6.49082661 \cdot 10^{-3}$ & 0.063 \\
$2^{-9}$ & $2^{-5}$ & $3.35139036 \cdot 10^{-3}$ & 0.502 \\
$2^{-10}$ & $2^{-6}$ & $1.70254707 \cdot 10^{-3}$ & 4.039 \\
$2^{-11}$ & $2^{-7}$ & $8.58257280 \cdot 10^{-4}$ & 32.574 \\
\hline
\end{tabular}

Table 2. Explicit Euler method, violated CFL condition

\begin{tabular}{ccc}
\hline$h_{0}$ & $h_{1}=h_{2}$ & Maximal error \\
\hline $2^{-6}$ & $2^{-4}$ & $2.31260490 \cdot 10^{0}$ \\
$2^{-7}$ & $2^{-5}$ & $7.77142720 \cdot 10^{7}$ \\
$2^{-8}$ & $2^{-6}$ & $5.43515795 \cdot 10^{21}$ \\
$2^{-9}$ & $2^{-7}$ & $+\infty$ \\
\hline
\end{tabular}

Now we consider the implicit Euler method and the (CFL) condition is not satisfied.

Table 3. Errors for implicit Euler method

\begin{tabular}{cccc}
\hline$h_{0}$ & $h_{1}=h_{2}$ & Maximal error & Time $[\mathrm{s}]$ \\
\hline $2^{-8}$ & $2^{-6}$ & $6.11531734 \cdot 10^{-3}$ & 0.982 \\
$2^{-9}$ & $2^{-7}$ & $3.10254097 \cdot 10^{-3}$ & 8.194 \\
$2^{-10}$ & $2^{-8}$ & $1.56092644 \cdot 10^{-3}$ & 63.151 \\
$2^{-11}$ & $2^{-9}$ & $8.04424286 \cdot 10^{-4}$ & 510.758 \\
\hline
\end{tabular}


Consider the differential integral equation

$$
\begin{aligned}
\partial_{t} z(t, x, y)= & 4 x\left\{1+\sin \left[2 t \int_{-1}^{x} s z(t, s, y) d s-z(t, x, y)\right]\right\} \partial_{x} z(t, x, y) \\
& +4 y\left\{1-\sin \left[2 t \int_{-1}^{y} s z(t, x, s) d s-z(t, x, y)\right]\right\} \partial_{y} z(t, x, y) \\
= & f(t, x, y) z(t, x, y)
\end{aligned}
$$

with the initial boundary conditions (52)-54) where

$f(t, x, y)=x^{2}+y^{2}-1-8 t\left(x^{2}+y^{2}\right)+8 t x^{2} \sin \left[\exp \left(t y^{2}\right)\right]-8 t y^{2} \sin \left[\exp \left(t x^{2}\right)\right]$.

The solution of the above problem is $v(t, x, y)=\exp \left[t\left(x^{2}+y^{2}-1\right)\right]$. The following tables show maximal error values for several step sizes.

Table 4. Errors for explicit Euler method

\begin{tabular}{cccc}
\hline$h_{0}$ & $h_{1}=h_{2}$ & Maximal error & Time [s] \\
\hline $2^{-8}$ & $2^{-4}$ & $6.63816929 \cdot 10^{-3}$ & 0.063 \\
$2^{-9}$ & $2^{-5}$ & $3.43430042 \cdot 10^{-3}$ & 0.502 \\
$2^{-10}$ & $2^{-6}$ & $1.74641609 \cdot 10^{-3}$ & 4.039 \\
$2^{-11}$ & $2^{-7}$ & $8.80718231 \cdot 10^{-4}$ & 32.574 \\
\hline
\end{tabular}

Table 5. Explicit Euler method, violated CFL condition

\begin{tabular}{ccc}
\hline$h_{0}$ & $h_{1}=h_{2}$ & Maximal error \\
\hline $2^{-6}$ & $2^{-4}$ & $4.39485836 \cdot 10^{0}$ \\
$2^{-7}$ & $2^{-5}$ & $1.76221740 \cdot 10^{7}$ \\
$2^{-8}$ & $2^{-6}$ & $1.99352761 \cdot 10^{19}$ \\
$2^{-9}$ & $2^{-7}$ & $+\infty$ \\
\hline
\end{tabular}

Now we consider the implicit Euler method and the (CFL) condition is not satisfied.

Table 6. Errors for implicit Euler method

\begin{tabular}{cccc}
\hline$h_{0}$ & $h_{1}=h_{2}$ & Maximal error & Time $[\mathrm{s}]$ \\
\hline $2^{-8}$ & $2^{-6}$ & $6.07287884 \cdot 10^{-3}$ & 1.353 \\
$2^{-9}$ & $2^{-7}$ & $3.11011076 \cdot 10^{-3}$ & 11.014 \\
$2^{-10}$ & $2^{-8}$ & $1.57845020 \cdot 10^{-3}$ & 88.759 \\
$2^{-11}$ & $2^{-9}$ & $7.96794891 \cdot 10^{-4}$ & 715.743 \\
\hline
\end{tabular}

Our considerations reveal the following relations between explicit and implicit difference methods for (1), (2). Assumptions on the regularity of given functions are the same in the theorems on convergence of explicit and 
implicit difference schemes. We need condition (46) on the mesh for explicit difference methods, but not for implicit methods. Error estimates are the same for both methods. Tables 2, 3 and 5,6 show that there are implicit difference methods which are convergent, while the corresponding explicit schemes are not.

4. Parabolic functional differential equations. We formulate difference methods for (3), (2). Consider the operator $W_{h}: \Omega_{h} \times F\left(A_{h}, \mathbb{R}\right) \rightarrow \mathbb{R}$ defined in the following way. Let $\left(t^{(r)}, x^{(m)}, w, \zeta\right) \in \Omega_{h} \times F\left(A_{h}, \mathbb{R}\right)$. Write

$$
\begin{aligned}
& S_{+}^{(r, m)}[w]=\left\{(i, j): 1 \leq i, j \leq n, i \neq j, F_{i j}\left(t^{(r)}, x^{(m)}, w\right) \geq 0\right\}, \\
& S_{-}^{(r, m)}[w]=\left\{(i, j): 1 \leq i, j \leq n, i \neq j, F_{i j}\left(t^{(r)}, x^{(m)}, w\right)<0\right\},
\end{aligned}
$$

and

$$
\delta_{i}^{+} \zeta^{(\theta)}=\frac{1}{h_{i}}\left[\zeta^{\left(e_{i}\right)}-\zeta^{(\theta)}\right], \quad \delta_{i}^{-} \zeta^{(\theta)}=\frac{1}{h_{i}}\left[\zeta^{(\theta)}-\zeta^{\left(-e_{i}\right)}\right], \quad 1 \leq i \leq n .
$$

Set

$$
\begin{aligned}
W_{h}\left(t^{(r)}, x^{(m)}, w, \zeta\right)= & \sum_{i, j=1}^{n} F_{i j}\left(t^{(r)}, x^{(m)}, w\right) \delta_{i j} \zeta^{(\theta)} \\
& +\sum_{i=1}^{n} G_{i}\left(t^{(r)}, x^{(m)}, w\right) \delta_{i} \zeta^{(\theta)}+G\left(t^{(r)}, x^{(m)}, w\right) .
\end{aligned}
$$

The expressions $\delta \zeta^{(\theta)}=\left(\delta_{1} \zeta^{(\theta)}, \ldots, \delta_{n} \zeta^{(\theta)}\right)$ and $\delta^{(2)} \zeta^{(\theta)}=\left[\delta_{i j} \zeta^{(\theta)}\right]_{i, j=1}^{n}$ are defined in the following way:

$$
\delta_{i} \zeta^{(\theta)}=\frac{1}{2 h_{i}}\left[\zeta^{\left(e_{i}\right)}-\zeta^{\left(-e_{i}\right)}\right], \quad \delta_{i i} \zeta^{(\theta)}=\delta_{i}^{+} \delta_{i}^{-} \zeta^{(\theta)} \quad \text { for } i=1, \ldots, n .
$$

and

$$
\delta_{i j} \zeta^{(\theta)}= \begin{cases}\frac{1}{2}\left[\delta_{i}^{+} \delta_{j}^{+} \zeta^{(\theta)}+\delta_{i}^{-} \delta_{j}^{-} \zeta^{(\theta)}\right] & \text { for }(i, j) \in S_{+}^{(r, m)}[w], \\ \frac{1}{2}\left[\delta_{i}^{+} \delta_{j}^{-} \zeta^{(\theta)}+\delta_{i}^{-} \delta_{j}^{+} \zeta^{(\theta)}\right] & \text { for }(i, j) \in S_{-}^{(r, m)}[w] .\end{cases}
$$

For $z \in F\left(E_{0 . h} \cup E_{h}\right)$ and $\left(t^{(r)}, x^{(m)}\right) \in E_{h}^{\prime}$ we put

$$
\begin{aligned}
\mathbb{W}_{\text {ex. } h}[z]^{(r, m)} & =W_{h}\left(t^{(r)}, x^{(m)},\left(T_{h} z\right)_{[r, m]}, z_{\langle r, m\rangle}\right), \\
\mathbb{W}_{\text {im. } h}[z]^{(r, m)} & =W_{h}\left(t^{(r)}, x^{(m)},\left(T_{h} z\right)_{[r, m]}, z_{\langle r+1, m\rangle}\right) .
\end{aligned}
$$

Given $\varphi_{h}: E_{0 . h} \cup \partial_{0} E_{h} \rightarrow \mathbb{R}$, we approximate classical solutions to (3), (2) with solutions of the functional difference equation

$$
\delta_{0} z^{(r, m)}=\mathbb{W}_{\text {ex.h }}[z]^{(r, m)}
$$

with the initial boundary condition

$$
z^{(r, m)}=\varphi_{h}^{(r, m)} \quad \text { on } E_{0 . h} \cup \partial_{0} E_{h} .
$$


The functional difference equation

$$
\delta_{0} z^{(r, m)}=\mathbb{W}_{\operatorname{im} . h}[z]^{(r, m)}
$$

with the initial boundary condition (57) is considered to be an implicit difference scheme for (3), (2).

We first construct estimates of solutions to (3), (2). A function $z \in C\left(E_{0} \cup\right.$ $E, \mathbb{R})$ will be called of class $C^{1.2}$ if $z(\cdot, x):\left[-b_{0}, a\right] \rightarrow \mathbb{R}$ is of class $C^{1}$ for $x \in[-b, b]$ and $z(t, \cdot):[-b, b] \rightarrow \mathbb{R}$ is of class $C^{2}$ for $t \in\left[-b_{0}, a\right]$.

Assumption $H_{0}[\mathbf{F}, \varphi]$. The functions $\mathbf{F}: \Xi \rightarrow M_{n \times n}$ and $\mathbf{G}: \Xi \rightarrow \mathbb{R}^{n}$, $G: \Xi \rightarrow \mathbb{R}$ are continuous and they satisfy the condition $(V)$ and

1) the matrix $\mathbf{F}$ is symmetric and for $(t, x, w) \in \Xi$ we have

$$
\sum_{i, j=1}^{n} F_{i j}(t, x, w) y_{i} y_{j} \geq 0, \quad y=\left(y_{1}, \ldots, y_{n}\right) \in \mathbb{R}^{n},
$$

2) there is $\varrho:[0, a] \times \mathbb{R}_{+} \rightarrow \mathbb{R}_{+}$such that Assumption $H[\varrho]$ is satisfied and

$$
|G(t, x, w)| \leq \varrho\left(t,\|w\|_{B}\right) \quad \text { on } \Xi
$$

3) $\varphi \in C\left(E_{0} \cup \partial_{0} E, \mathbb{R}\right), \varphi_{h} \in \mathbf{F}\left(E_{0 . h} \cup \partial_{0} E_{h}, \mathbb{R}\right)$ and there is $\alpha_{0}: H \rightarrow \mathbb{R}_{+}$ such that

$$
\left|\varphi(t, x)-\varphi_{h}(t, x)\right| \leq \alpha_{0}(h) \quad \text { on } E_{0 . h} \cup \partial_{0} E_{h} \quad \text { and } \quad \lim _{h \rightarrow 0} \alpha_{0}(h)=0,
$$

4) $\tilde{\eta} \in \mathbb{R}_{+}$is such that

$$
\begin{aligned}
|\varphi(t, x)| \leq \tilde{\eta} \quad \text { on } E_{0} \quad \text { and } \quad\left|\varphi_{h}(t, x)\right| \leq \tilde{\eta} \quad \text { on } E_{0 . h}, \\
|\varphi(t, x)| \leq \omega(t, \tilde{\eta}) \quad \text { on } \partial_{0} E \quad \text { and } \quad\left|\varphi_{h}(t, x)\right| \leq \omega(t, \tilde{\eta}) \quad \text { on } \partial_{0} E_{h},
\end{aligned}
$$

where $\omega(\cdot \tilde{\eta})$ is the maximal solution to 13 with $\eta=\tilde{\eta}$.

Lemma 4.1. If Assumption $H_{0}[\mathbf{F}, \varphi]$ is satisfied and $\tilde{z}: E_{0} \cup E \rightarrow \mathbb{R}$ is a solution to (3), (2) and $\tilde{z}$ is of class $C^{1.2}$ then

$$
|\tilde{z}(t, x)| \leq \omega(t, \tilde{\eta}) \quad \text { on } E .
$$

Proof. For $\varepsilon>0$ we denote by $\omega(\cdot, \tilde{\eta}, \varepsilon)$ the maximal solution of the Cauchy problem $(13)$. The function $\omega(\cdot, \tilde{\eta}, \varepsilon)$ is defined on $[0, a]$ and it satisfies condition (43). Write $\tilde{\omega}(t)=\|\tilde{z}\|_{t}, t \in[0, a]$. We prove that

$$
\tilde{\omega}(t)<\omega(t, \tilde{\eta}, \varepsilon) \quad \text { for } t \in[0, a] .
$$

Suppose for contradiction that assertion 62 fails to be true. Then there exists $(\tilde{t}, \tilde{x}) \in(0, a] \times(-b, b)$ such that

$$
\tilde{\omega}(t)<\omega(t, \tilde{\eta}, \varepsilon) \quad \text { for } t \in[0, \tilde{t}), \quad \tilde{\omega}(\tilde{t})=\omega(\tilde{t}, \tilde{\eta}, \varepsilon)=|\tilde{z}(\tilde{t}, \tilde{x})| .
$$

Suppose that $\tilde{\omega}(\tilde{t})=\tilde{z}(\tilde{t}, \tilde{x})$. Then

$$
D_{-} \tilde{\omega}(\tilde{t}) \geq \omega^{\prime}(\tilde{t}, \tilde{\eta}, \varepsilon) .
$$


Moreover $\partial_{x} \tilde{z}(\tilde{t}, \tilde{x})=\theta$ and

$$
\sum_{i, j=1}^{n} \partial_{x_{i} x_{j}} \tilde{z}(\tilde{t}, \tilde{x}) y_{i} y_{j} \leq 0 \quad \text { for } y=\left(y_{1}, \ldots, y_{n}\right) \in \mathbb{R}^{n} .
$$

We conclude from $(59), 60), 64$ that

$$
D_{-} \tilde{\omega}(\tilde{t}) \leq \partial_{t} \tilde{z}(\tilde{t}, \tilde{x}) \leq G\left(\tilde{t}, \tilde{x}, \tilde{z}_{(\tilde{t}, \tilde{x})}\right) \leq \varrho(\tilde{t}, \omega(\tilde{t}, \tilde{\eta}, \varepsilon))<\omega^{\prime}(\tilde{t}, \tilde{\eta}, \varepsilon),
$$

which contradicts $(62)$. The case $\tilde{\omega}(\tilde{t})=-\tilde{z}(\tilde{t}, \tilde{x})$ can be treated in a similar way. Thus inequality 62 is proved. Letting $\varepsilon$ tend to zero in $(62)$ we obtain 61.

Assumption $H[\mathbf{F}, \varphi]$. Assumption $H_{0}[\mathbf{F}, \varphi]$ is satisfied and the steps of the mesh satisfy

$$
\begin{aligned}
\frac{1}{h_{i}} F_{i i}(P)-\sum_{\substack{j=1 \\
j \neq i}}^{n} \frac{1}{h_{j}}\left|F_{i j}(P)\right|-\frac{1}{2}\left|G_{i}(P)\right| & \geq 0, \\
P & =(t, x, w) \in \Xi, i=1, \ldots, n .
\end{aligned}
$$

REMARK 4.2. Suppose that there is $\tilde{a}>0$ such that

$$
F_{i i}(P)-\sum_{\substack{j=1 \\ j \neq i}}^{n}\left|F_{i j}(P)\right| \geq \tilde{a}, \quad P \in \Xi, i=1, \ldots, n .
$$

Then condition (59) is satisfied (see [19]) and there is $\varepsilon_{0}>0$ such that for $\left\|h^{\prime}\right\|<\varepsilon_{0}$ and for $h_{1}=h_{2}, \ldots, h_{n}$ inequalities (65) hold.

Lemma 4.3. Suppose that Assumptions $H\left[T_{h}\right]$ and $H[\mathbf{F}, \varphi]$ are satisfied. Then:

I. There exists exactly one solution $v_{h}: E_{0 . h} \cup E_{h} \rightarrow \mathbb{R}$ to (58), (57) and

$$
\left|v_{h}^{(r, m)}\right| \leq \omega\left(t^{(r)}, \bar{\eta}\right) \quad \text { on } E_{h} .
$$

\section{If additionally}

$$
1-2 h_{0} \sum_{i=1}^{n} \frac{1}{h_{i}^{2}} F_{i i}(P)+\sum_{\substack{i, j=1 \\ j \neq i}}^{n} \frac{1}{h_{j}}\left|F_{i j}(P)\right| \geq 0,
$$

where $P=(t, x, w) \in \Xi$, then the solution $u_{h}: E_{0 . h} \cup E_{h} \rightarrow \mathbb{R}$ to (56), (57) satisfies the condition

$$
\left|u_{h}^{(r, m)}\right| \leq \omega\left(t^{(r)}, \tilde{\eta}\right) \quad \text { on } E_{h} .
$$

Proof. We apply Theorem 2.1. Consider the functions $f_{h}: \Omega_{h} \rightarrow \mathbb{R}$ and $G_{h}: \Omega_{h} \rightarrow \mathbb{R}^{\chi}, G_{h}=\left\{G_{h . \lambda}\right\}_{\lambda \in \Lambda}$, defined in the following way. Let $\left(t^{(r)}, x^{(m)}, w\right) \in \Omega_{h}$. Write

$\Lambda_{0}^{(r, m)}[w]=\left\{\lambda \in \Lambda\right.$ : there is $i, 1 \leq i \leq n$, such that $\lambda=e_{i}$ or $\left.\lambda=-e_{i}\right\}$, 


$$
\begin{aligned}
& \Lambda_{I}^{(r, m)}[w]=\left\{\lambda \in \Lambda: \text { there is }(i, j) \in S_{+}^{(r, m)}[w]\right. \text { such that } \\
& \left.\quad \lambda=e_{i}+e_{j} \text { or } \lambda=-e_{i}-e_{j}\right\}, \\
& \Lambda_{I I}^{(r, m)}[w]=\left\{\lambda \in \Lambda: \text { there is }(i, j) \in S_{-}^{(r, m)}[w]\right. \text { such that } \\
& \left.\lambda=e_{i}-e_{j} \text { or } \lambda=-e_{i}+e_{j}\right\}, \\
& \tilde{\Lambda}^{(r, m)}[w]=\Lambda \backslash\left\{\Lambda_{0}^{(r, m)}[w] \cup \Lambda_{I}^{(r, m)}[w] \cup \Lambda_{I I}^{(r, m)}[w] \cup\{\theta\}\right\} .
\end{aligned}
$$

Set $P=(t, x, w) \in \Omega$ and

$$
\begin{aligned}
f_{h}(P) & =G(P), \quad G_{h . \theta}(P)=-2 \sum_{i=1}^{n} \frac{1}{h_{i}^{2}} F_{i i}(P)+\sum_{\substack{i, j=1 \\
i \neq j}}^{n} \frac{1}{h_{i} h_{j}}\left|F_{i j}(P)\right|, \\
G_{h . e_{i}}(P) & =\frac{1}{h_{i}^{2}} F_{i i}(P)-\sum_{\substack{j=1 \\
j \neq i}}^{n} \frac{1}{h_{i} h_{j}}\left|F_{i j}(P)\right|+\frac{1}{2 h_{i}} G_{i}(P), \\
G_{h .-e_{i}}(P) & =\frac{1}{h_{i}^{2}} F_{i i}(P)-\sum_{\substack{j=1 \\
j \neq i}}^{n} \frac{1}{h_{i} h_{j}}\left|F_{i j}(P)\right|-\frac{1}{2 h_{i}} G_{i}(P), \\
G_{h . e_{i}+e_{j}}(P) & =G_{h .-e_{i}-e_{j}}(P)=-\frac{1}{2 h_{i} h_{j}} F_{i j}(P), \quad(i, j) \in S_{+}^{(r, m)}[w], \\
G_{h . e_{i}-e_{j}}(P) & =G_{h .-e_{i}+e_{j}}(P)=\frac{1}{2 h_{i} h_{j}} F_{i j}(P), \quad(i, j) \in S_{-}^{(r, m)}[w], \\
G_{h . \lambda}(P) & =0 \quad \text { for } \lambda \in \tilde{\Lambda}[w]^{(r, m)} .
\end{aligned}
$$

Then Assumption $H\left[G_{h}, f_{h}\right]$ is satisfied and problems (56), (57) and (58), (57) are equivalent to (6), (7) and (12), (7) respectively. Our lemma follows from Theorem 2.1.

Suppose that Assumptions $H\left[T_{h}\right]$ and $H[\mathbf{F}, \varphi]$ are satisfied. Write $\tilde{C}=$ $\omega(a, \tilde{\eta})$. Let $X[\tilde{C}]$ be defined by 47$]$.

Assumption $H[\mathbf{F}, \sigma]$. The functions $\mathbf{F}: \Xi \rightarrow M_{n \times n}, \mathbf{G}: \Xi \rightarrow \mathbb{R}^{n}$, $G: \Xi \rightarrow \mathbb{R}$ and $\varphi: E_{0} \cup \partial_{0} E \rightarrow \mathbb{R}$ satisfy Assumption $H_{0}[\mathbf{F}, \varphi]$ and there is $\sigma:[0, a] \times \mathbb{R}_{+}$such that Assumption $H[\sigma]$ holds and the expressions $\|\mathbf{F}(t, x, w)-\mathbf{F}(t, x, \tilde{w})\|,\|\mathbf{G}(t, x, w)-\mathbf{G}(t, x, \tilde{w})\|,|G(t, x, w)-G(t, x, \tilde{w})|$ are bounded from above by $\sigma\left(t,\|w-\tilde{w}\|_{B}\right)$ for all $(t, x) \in E$ and $w, \tilde{w} \in X[\tilde{C}]$.

REMARK 4.4. It is important that we have assumed nonlinear estimates for $w, \tilde{w} \in X[\tilde{C}]$. It is clear that there are differential integral equations and differential equations with deviated variables such that Assumption $H[\mathbf{F}, \sigma]$ holds and the nonlinear estimates are not satisfied on $\Xi$. 
TheOREM 4.5. Suppose that Assumptions $H\left[T_{h}\right]$ and $H[\mathbf{F}, \sigma]$ are satisfied and $\tilde{z}: E_{0} \cup E \rightarrow \mathbb{R}$ is a solution to (3), (2) of class $C^{1.2}$, and $\tilde{z}_{h}$ is the restriction of $\tilde{z}$ to the set $E_{0 . h} \cup E_{h}$. Then:

I. There exists exactly one solution $v_{h}: E_{0 . h} \cup E_{h} \rightarrow \mathbb{R}$ to (58), (57) and there is $\alpha: H \rightarrow \mathbb{R}_{+}$such that

$$
\left|\left(v_{h}-\tilde{z}_{h}\right)^{(r, m)}\right| \leq \alpha(h) \quad \text { on } E_{h} \quad \text { and } \quad \lim _{h \rightarrow 0} \alpha(h)=0 .
$$

II. If condition (66) is satisfied then there is $\alpha: H \rightarrow \mathbb{R}_{+}$such that

$$
\left|\left(u_{h}-\tilde{z}_{h}\right)^{(r, m)}\right| \leq \alpha(h) \quad \text { on } E_{h} \quad \text { and } \quad \lim _{h \rightarrow 0} \alpha(h)=0,
$$

where $u_{h}: E_{0 . h} \cup E_{h} \rightarrow \mathbb{R}_{+}$is a solution to (56), (57).

Proof. We apply Theorem 2.2 to prove (67), (68). Let $\tilde{c} \in \mathbb{R}_{+}$be such that

$$
\left\|\partial_{x} \tilde{z}(t, x)\right\| \leq \tilde{c}, \quad\left\|\partial_{x x} \tilde{z}(t, x)\right\| \leq \tilde{c} \quad \text { for }(t, x) \in E .
$$

Denote by $Y_{h}$ the class of all $\zeta \in \mathbf{F}\left(A_{h}, \mathbb{R}\right)$ satisfying

$$
\begin{gathered}
\frac{1}{2}\left|\delta_{i}^{+} \zeta^{(\theta)}+\delta_{i}^{(\theta)}\right| \leq \tilde{c}, \quad i=1, \ldots, n, \\
\frac{1}{2}\left|\delta_{i}^{+} \delta_{j}^{+} \zeta^{(\theta)}+\delta_{i}^{-} \delta_{j}^{-} \zeta^{(\theta)}\right| \leq \tilde{c}, \quad \frac{1}{2}\left|\delta_{i}^{+} \delta_{j}^{-} \zeta^{(\theta)}+\delta_{i}^{-} \delta_{j}^{+} \zeta^{(\theta)}\right| \leq \tilde{c}, \quad i, j=1, \ldots, n .
\end{gathered}
$$

Then

$$
\left\|\tilde{z}_{h}\right\|_{h . r} \leq \tilde{C}, \quad\left(\tilde{z}_{h}\right)_{\langle r, m\rangle} \in Y_{h} \quad \text { for } r=0,1, \ldots, K .
$$

It follows from Assumptions $H\left[T_{h}\right]$ and $H[\mathbf{F}, \sigma]$ that there is $\gamma: H \rightarrow \mathbb{R}_{+}$ such that

$$
\begin{aligned}
\left|\delta_{0} \tilde{z}_{h}^{(r, m)}-\mathbb{W}_{\text {im.h }}\left[\tilde{z}_{h}\right]^{(r, m)}\right| \leq \gamma(h) & \text { on } E_{h}^{\prime}, \\
\left|\delta_{0} \tilde{z}_{h}^{(r, m)}-\mathbb{W}_{\text {ex.h }}\left[\tilde{z}_{h}\right]^{(r, m)}\right| \leq \gamma(h) & \text { on } E_{h}^{\prime} .
\end{aligned}
$$

We conclude from Assumption $H[\mathbf{F}, \sigma]$ that there is $\bar{c}>0$ such that the operator $W_{h}$ given by (55) satisfies

$$
\left|W_{h}(t, x, w, \zeta)-W_{h}(t, x, \tilde{w}, \zeta)\right| \leq(1+\bar{c}) \sigma\left(t,\|w-\tilde{w}\|_{B}\right)
$$

for all $(t, x) \in E_{h}^{\prime}, w, \tilde{w} \in X[\tilde{C}]$ and $\zeta \in Y_{h}$. It is clear that condition 66 for equation (56) is equivalent to (15) for equation (6). Thus all the assumptions of Theorem 2.2 are satisfied and assertions 67, 68 follow.

REMARK 4.6. Suppose that all the assumptions of Theorem 4.5 are satisfied with $\sigma(t, p)=\tilde{L} p$ on $[0, a] \times \mathbb{R}_{+}$where $\tilde{L} \in \mathbb{R}_{+}$. Then there is $L \in \mathbb{R}_{+}$ such that $\left|\left(\tilde{z}_{h}-v_{h}\right)^{(r, m)}\right| \leq \tilde{\alpha}(h)$ on $E_{h}$ and $\left|\left(\tilde{z}_{h}-u_{h}\right)^{(r, m)}\right| \leq \tilde{\alpha}(h)$ on $E_{h}$ where $\tilde{\alpha}: H \rightarrow \mathbb{R}_{+}$is given by (34), (35).

Now we give numerical examples. Put $n=2$. Solutions of the initial boundary value problems are defined on $E=[0,0.5] \times[-1,1] \times[-1,1]$. 
Consider the differential equation with deviated variables

$$
\begin{aligned}
\partial_{t} z(t, x, y)=\{ & \left.2+\cos \left[z(t, 0.5(x+y), 0.5(x-y))-e^{t x y}\right]\right\} \partial_{x x} z(t, x, y) \\
& +\left\{2+\cos \left[z(t, 0.5(x-y), 0.5(x+y))-e^{-t x y}\right]\right\} \partial_{y y} z(t, x, y) \\
& +\partial_{x y} z(t, x, y) \sin [z(0.25 t, x, y)-z(t, 0.5 x, 0.5 y)] \\
& +f(t, x, y) z(t, x, y), \\
f(t, x, y)= & x^{2}-y^{2}-12 t^{2}\left(x^{2}+y^{2}\right),
\end{aligned}
$$

with the initial boundary conditions

$$
\begin{aligned}
z(0, x, y) & =1, \quad(x, y) \in[-1,1] \times[-1,1], \\
z(t,-1, y) & =z(t, 1, y)=e^{t\left(1-y^{2}\right)}, \quad t \in[0,0.5], y \in[-1,1], \\
z(t, x,-1) & =z(t, x, 1)=e^{t\left(x^{2}-1\right)}, \quad t \in[0,0.5], x \in[-1,1] .
\end{aligned}
$$

The solution of the above problem is $v(t, x, y)=\exp \left[t\left(x^{2}-y^{2}\right)\right]$. The following tables show maximal values of errors for several step sizes.

Table 7. Errors for explicit Euler method

\begin{tabular}{cccc}
\hline$h_{0}$ & $h_{1}=h_{2}$ & Maximal error & Time [s] \\
\hline $2^{-8}$ & $2^{-1}$ & $6.09265984 \cdot 10^{-3}$ & 0.115 \\
$2^{-10}$ & $2^{-3}$ & $3.10700168 \cdot 10^{-3}$ & 1.142 \\
$2^{-12}$ & $2^{-4}$ & $1.56094035 \cdot 10^{-3}$ & 19.312 \\
$2^{-14}$ & $2^{-5}$ & $7.81394600 \cdot 10^{-4}$ & 318.221 \\
\hline
\end{tabular}

Table 8. Errors for explicit Euler scheme, violated CFL condition

\begin{tabular}{ccc}
\hline$h_{0}$ & $h_{1}=h_{2}$ & Maximal error \\
\hline $2^{-5}$ & $2^{-2}$ & $3.80077434 \cdot 10^{1}$ \\
$2^{-7}$ & $2^{-3}$ & $3.37466385 \cdot 10^{17}$ \\
$2^{-9}$ & $2^{-4}$ & $7.36222251 \cdot 10^{88}$ \\
$2^{-11}$ & $2^{-5}$ & $+\infty$ \\
\hline
\end{tabular}

Now we consider the implicit Euler method with steps of the mesh given in Table 8.

Table 9. Errors for implicit Euler scheme

\begin{tabular}{cccc}
\hline$h_{0}$ & $h_{1}=h_{2}$ & Maximal error & Time $[\mathrm{s}]$ \\
\hline $2^{-5}$ & $2^{-2}$ & $2.70180118 \cdot 10^{-2}$ & 0.112 \\
$2^{-7}$ & $2^{-3}$ & $1.46550838 \cdot 10^{-2}$ & 1.251 \\
$2^{-9}$ & $2^{-4}$ & $7.47607123 \cdot 10^{-3}$ & 24.920 \\
$2^{-11}$ & $2^{-5}$ & $3.75674966 \cdot 10^{-3}$ & 941.878 \\
\hline
\end{tabular}


Consider the differential integral equation

$$
\begin{aligned}
\partial_{t} z(t, x, y)= & \left\{2+\cos \left[2 t \int_{0}^{x} s z(t, s, y) d s-z(t, x, y)\right]\right\} \partial_{x x} z(t, x, y) \\
& +\left\{2+\cos \left[2 t \int_{0}^{y} s z(t, x, s) d s+z(t, x, y)\right]\right\} \partial_{y y} z(t, x, y) \\
& +\partial_{x y} z(t, x, y) \sin \left[1+\left(x^{2}-y^{2}\right) \int_{0}^{t} z(\tau, x, y) d \tau-z(t, x, y)\right] \\
& +f(t, x, y) z(t, x, y)
\end{aligned}
$$

with initial boundary conditions (69)-(71) where

$$
\begin{aligned}
f(t, x, y))= & x^{2}-y^{2}-8 t^{2}\left(x^{2}+y^{2}\right)-2 t\left(1+2 x^{2} t\right) \cos e^{-t y^{2}} \\
& -2 t\left(-1+2 y^{2} t\right) \cos e^{t x^{2}} .
\end{aligned}
$$

The function $v(t, x, y)=e^{t\left(x^{2}-y^{2}\right)}$ is a solution of the above problem. The following tables show maximal values of errors for several step sizes.

Table 10. Errors for explicit Euler method

\begin{tabular}{cccc}
\hline$h_{0}$ & $h_{1}=h_{2}$ & Maximal error & Time $[\mathrm{s}]$ \\
\hline $2^{-8}$ & $2^{-1}$ & $5.63773239 \cdot 10^{-3}$ & 0.006 \\
$2^{-10}$ & $2^{-3}$ & $2.87933980 \cdot 10^{-3}$ & 0.052 \\
$2^{-12}$ & $2^{-4}$ & $1.44708568 \cdot 10^{-3}$ & 0.766 \\
$2^{-14}$ & $2^{-5}$ & $7.24464180 \cdot 10^{-4}$ & 12.727 \\
\hline
\end{tabular}

Table 11. Errors for explicit Euler scheme, violated CFL condition

\begin{tabular}{ccc}
\hline$h_{0}$ & $h_{1}=h_{2}$ & Maximal error \\
\hline $2^{-5}$ & $2^{-2}$ & $9.57986240 \cdot 10^{0}$ \\
$2^{-7}$ & $2^{-3}$ & $6.01045114 \cdot 10^{16}$ \\
$2^{-9}$ & $2^{-4}$ & $2.40913798 \cdot 10^{87}$ \\
$2^{-11}$ & $2^{-5}$ & $+\infty$ \\
\hline
\end{tabular}

Now we consider the implicit Euler method with steps of the mesh given in Table 11.

Table 12. Errors for implicit Euler scheme

\begin{tabular}{cccc}
\hline$h_{0}$ & $h_{1}=h_{2}$ & Maximal error & Time $[\mathrm{s}]$ \\
\hline $2^{-5}$ & $2^{-2}$ & $2.69819681 \cdot 10^{-2}$ & 0.938 \\
$2^{-7}$ & $2^{-3}$ & $1.47067642 \cdot 10^{-2}$ & 0.827 \\
$2^{-9}$ & $2^{-4}$ & $7.51138801 \cdot 10^{-3}$ & 22.291 \\
$2^{-11}$ & $2^{-5}$ & $3.77561536 \cdot 10^{-3}$ & 899.428 \\
\hline
\end{tabular}


Our considerations show the following relations between explicit and implicit difference methods for (3), (2). Assumptions on the regularity of given functions are the same in the theorems on convergence of explicit and implicit difference schemes. We need condition (46) on the mesh for explicit difference methods, but not for implicit ones. Error estimates are the same for both methods. Tables 8, 9 and 11, 12 show that there are implicit difference methods which are convergent, while the corresponding explicit schemes are not.

\section{References}

[1] A. Baranowska, Numerical methods for nonlinear first-order partial differential equations with deviated variables, Numer. Methods Partial Differential Equations 22 (2006), 708-727.

[2] H. Brunner, The numerical treatment of ordinary and partial Volterra integrodifferential equations, in: Proc. First Internat. Colloq. Numer. Anal. (Plovdiv, 1992), D. Bainov (ed.), VSP, Utrecht, 1993, 13-26.

[3] R. Ciarski, Stability of difference equations generated by quasilinear differential functional problems, Demonstratio Math. 35 (2002), 557-571.

[4] - Numerical approximations of parabolic differential functional equations with the initial boundary conditions of the Neumann type, Ann. Polon. Math. 84 (2004), 103-119.

[5] W. Czernous and Z. Kamont, Implicit difference methods for parabolic functional differential equations, Z. Angew. Math. Mech. 85 (2005), 326-338.

[6] E. Godlewski and P. Raviart, Numerical Approximation of Hyperbolic Systems of Conservation Laws, Springer, Berlin, 1996.

[7] Z. Kamont, Hyperbolic Functional Differential Inequalities and Applications, Kluwer, Dordrecht, 1999.

[8] Z. Kamont and H. Leszczyński, Stability of difference equations generated by parabolic differential functional equations, Rend. Mat. Appl. (7) 16 (1996), 265-287.

[9] A. Kępczyńska, Implicit difference methods for quasilinear differential functional equations on the Haar pyramid, Z. Anal. Anwend. 27 (2008), 213-231.

[10] K. Kropielnicka, Implicit difference methods for quasilinear parabolic functional differential problems of the Dirichlet type, Appl. Math. (Warsaw) 35 (2008), 155-175.

[11] X. Lu, Monotone method and convergence acceleration for finite-difference solutions of parabolic problems with time delays, Numer. Methods Partial Differential Equations 11 (1995), 591-602.

[12] —, Combined iterative methods for numerical solutions of parabolic problems with time delays, Appl. Math. Comput. 89 (1998), 213-224.

[13] M. Malec et A. Schiaffino, Méthode aux différences finies pour une équation nonlinéaire différentielle fonctionnelle du type parabolique avec une condition initiale de Cauchy, Boll. Un. Mat. Ital. B (7) 1 (1987), 99-109.

[14] C. V. Pao, Finite difference solutions of reaction diffusion equations with continuous time delays, Comput. Math. Appl. 42 (2001), 399-412.

[15] - Finite difference reaction-diffusion systems with coupled boundary conditions and time delays, J. Math. Anal. Appl. 272 (2002), 407-434.

[16] A. A. Samarskii, The Theory of Difference Schemes, Dekker, New York, 2001. 
[17] A. A. Samarskii, P. P. Matus and P. N. Vabishchevich, Difference Schemes with Operator Factors, Kluwer, Dordrecht, 2002.

[18] L. Sapa, A finite difference method for quasi-linear and nonlinear differential functional parabolic equations with Dirichlet's condition, Ann. Polon. Math. 93 (2008), 113-133.

[19] W. Voigt, Finite-difference schemes for parabolic problems with first and mixed second order derivatives, Z. Angew. Math. Mech. 68 (1988), 281-288.

[20] Y. M. Wang and C. V. Pao, Time delayed finite difference reaction-diffusion systems with nonquasimonotone functions, Numer. Math. 103 (2006), 485-513.

W. Czernous, Z. Kamont

Institute of Mathematics

University of Gdańsk

Wit Stwosz St. 57

80-952 Gdańsk, Poland

E-mail: wojciech.czernous@math.univ.gda.pl

zkamont@math.univ.gda.pl

Received on 26.10.2010

(2063) 